\title{
Isospin-dependent properties of asymmetric nuclear matter in relativistic mean field models
}

\author{
Lie-Wen Chen, ${ }^{1,2}$ Che Ming Ko, ${ }^{3}$ and Bao-An $\mathrm{Li}^{4}$ \\ ${ }^{1}$ Institute of Theoretical Physics, Shanghai Jiao Tong University, Shanghai 200240, People's Republic of China \\ ${ }^{2}$ Center of Theoretical Nuclear Physics, National Laboratory of Heavy Ion Accelerator, Lanzhou 730000, People's Republic of China \\ ${ }^{3}$ Cyclotron Institute and Physics Department, Texas A\&M University, College Station, Texas 77843-3366, USA \\ ${ }^{4}$ Department of Physics, Texas A\&M University-Commerce, Commerce, Texas 75429-3011, USA
}

(Received 6 September 2007; published 21 November 2007)

\begin{abstract}
Using various relativistic mean-field models, including nonlinear ones with meson field self-interactions, models with density-dependent meson-nucleon couplings, and point-coupling models without meson fields, we have studied the isospin-dependent bulk and single-particle properties of asymmetric nuclear matter. In particular, we have determined the density dependence of nuclear symmetry energy from these different relativistic mean-field models and compared the results with the constraints recently extracted from analyses of experimental data on isospin diffusion and isotopic scaling in intermediate energy heavy-ion collisions as well as from measured isotopic dependence of the giant monopole resonances in even- $A$ Sn isotopes. Among the 23 parameter sets in the relativistic mean-field model that are commonly used for nuclear structure studies, only a few are found to give symmetry energies that are consistent with the empirical constraints. We have also studied the nuclear symmetry potential and the isospin splitting of the nucleon effective mass in isospin asymmetric nuclear matter. We find that both the momentum dependence of the nuclear symmetry potential at fixed baryon density and the isospin splitting of the nucleon effective mass in neutron-rich nuclear matter depend not only on the nuclear interactions but also on the definition of the nucleon optical potential.
\end{abstract}

DOI: 10.1103/PhysRevC.76.054316

PACS number(s): 21.65.+f, 21.30.Fe, 24.10.Jv

\section{INTRODUCTION}

Besides the many existing radioactive beam facilities and their upgrades, many more are being constructed or planned, including the Cooling Storage Ring (CSR) facility at HIRFL in China [1], Radioactive Ion Beam (RIB) Factory at RIKEN in Japan [2], FAIR/GSI in Germany [3], SPIRAL2/GANIL in France [4], and Facility for Rare Isotope Beams (FRIB) in the U.S. [5]. These new facilities offer the possibility of studying the properties of nuclear matter or nuclei under the extreme condition of large isospin asymmetry. As a result, the study of the isospin degree of freedom in nuclear physics has recently attracted much attention. The ultimate goal of such study is to extract information on the isospin dependence of in-medium nuclear effective interactions as well as the equation of state (EOS) of isospin asymmetric nuclear matter, particularly its isospin-dependent term or the density dependence of the nuclear symmetry energy. This knowledge, especially the latter, is important for understanding not only the structure of radioactive nuclei, the reaction dynamics induced by rare isotopes, and the liquid-gas phase transition in asymmetric nuclear matter, but also many critical issues in astrophysics [6-13]. Unfortunately, the density dependence of the nuclear symmetry energy, especially its behavior at high densities, is largely unknown and is regarded as the most uncertain among all the properties of isospin asymmetric nuclear matter. Although the nuclear symmetry energy at normal nuclear matter density $\rho_{0} \approx 0.16 \mathrm{fm}^{-3}$ is known to be around $30 \mathrm{MeV}$ from the empirical liquid-drop mass formula [14,15], its values at other densities are poorly known [6,7]. Various microscopic and phenomenological models, such as the relativistic Dirac-Brueckner-Hartree-Fock (DBHF) [16-22] and nonrelativistic Brueckner-Hartree-Fock (BHF) $[23,24]$ approaches, the relativistic mean-field (RMF) model based on nucleon-meson interactions [12], and the nonrelativistic mean-field model based on Skyrme-like interactions [25-31], have been used to study the isospin-dependent properties of asymmetric nuclear matter, such as the nuclear symmetry energy, nuclear symmetry potential, and isospin splitting of nucleon effective mass, but the predicted results vary widely. In fact, even the sign of the symmetry energy above $3 \rho_{0}$ is uncertain [32]. The theoretical uncertainties are mainly due to the lack of knowledge about the isospin dependence of in-medium nuclear effective interactions and the limitations in the techniques for solving the nuclear manybody problem. As to the incompressibility of asymmetric nuclear matter, it is essentially undetermined [33], even after about 30 years of studies. For comparison, the incompressibility of symmetric nuclear matter at its saturation density $\rho_{0}$ has been determined to be $231 \pm 5 \mathrm{MeV}$ from the nuclear giant monopole resonances (GMR) [34], and the EOS at densities of $2 \rho_{0}<\rho<5 \rho_{0}$ has also been constrained by measurements of collective flows [8] and subthreshold kaon production [35] in nucleus-nucleus collisions.

As a phenomenological approach, the RMF model has achieved great success during the last decade in describing many nuclear phenomena [36-43]. For example, it provides a novel saturation mechanism for nuclear matter, an explanation of the strong spin-orbit interaction in finite nuclei, and a natural energy dependence of the nucleon optical potential. The RMF approach is generally based on effective interaction Lagrangians that involve nucleon and meson fields. In this approach, a number of parameters are adjusted to fit the properties of many nuclei. As such, these models usually give excellent descriptions of nuclear properties around or below the saturation density. 
Since the original Lagrangian proposed by Walecka more than 30 years ago [36], there have been a lot of different treatments, extensions, and applications of the RMF model. The three main versions are the nonlinear models [37-40], models with density-dependent meson-nucleon couplings [44-48], and point-coupling models without mesons [49-54]. For each version of the RMF model, there are also many different parameter sets with their values fitted to the binding energies and charge radii of a large number of nuclei in the periodic table. Including isovector mesons in the effective interaction Lagrangians further allows the RMF model to describe successfully the properties of nuclei far from the $\beta$-stability line. With recent developments in constraining the isospin-dependent properties of asymmetric nuclear matter, especially the density dependence of the nuclear symmetry energy, it is of great interest to see to what extent the results from different versions of the RMF model are consistent with these constrains.

In the present work, based on 23 different commonly used parameter sets in three different versions of the RMF model, we carry out a systematic study of the isospin-dependent bulk and single-particle properties of asymmetric nuclear matter. In particular, we study the density dependence of the nuclear symmetry energy from these RMF models and compare the results with the constraints recently extracted from analyses of the isospin diffusion data from heavy-ion collisions based on the isospin and momentum-dependent IBUU04 transport model with in-medium nucleon-nucleon $(N N)$ cross sections [55-57], isoscaling analyses of isotope ratios in intermediate energy heavy-ion collisions [58], and measured isotopic dependence of the GMR in even- $A$ Sn isotopes [59]. Among these 23 commonly used interactions in nuclear structure studies, only a few are found to give symmetry energies that are consistent with the empirically extracted one. Furthermore, we study the nuclear symmetry potential and the isospin splitting of the nucleon effective mass in isospin asymmetric nuclear matter. Our results indicate that the nuclear symmetry potential at fixed baryon density may increase or decrease with increasing nucleon momentum depending on the definition of the nucleon optical potential and the interactions used. This dependence is also seen in the isospin splitting of the nucleon effective mass in neutron-rich nuclear matter. In addition, the isospin splitting of the nucleon scalar density in neutron-rich nuclear matter is also studied.

The paper is organized as follows. In Sec. II, we discuss some isospin-dependent bulk and single-particle properties of asymmetric nuclear matter, such as the nuclear symmetry energy, nuclear symmetry potential, and isospin splitting of nucleon effective mass as well as current experimental and/or empirical constraints on these quantities. The theoretical frameworks for the different versions of RMF models, i.e., nonlinear RMF models, models with density-dependent nucleon-meson coupling, and nonlinear and density-dependent point-coupling models, are briefly reviewed in Sec. III. Results on the isospin-dependent properties of asymmetric nuclear matter, i.e., the nuclear symmetry energy, nuclear symmetry potential, and isospin splitting of nucleon effective mass and the nucleon scalar densities in neutron-rich nuclear matter, from different versions of RMF models are presented and discussed in
Sec. IV. A summary is then given in Sec. V. For completeness, the isospin- and momentum-dependent MDI interaction, which will be used as a reference in some cases for comparison, is briefly described in the Appendix.

\section{ISOSPIN-DEPENDENT PROPERTIES OF ASYMMETRIC NUCLEAR MATTER}

\section{A. Nuclear symmetry energy}

The EOS of isospin asymmetric nuclear matter, given by its binding energy per nucleon, can be generally written as

$$
E(\rho, \alpha)=E(\rho, \alpha=0)+E_{\text {sym }}(\rho) \alpha^{2}+O\left(\alpha^{4}\right),
$$

where $\rho=\rho_{n}+\rho_{p}$ is the baryon density, with $\rho_{n}$ and $\rho_{p}$ denoting the neutron and proton densities, respectively; $\alpha=$ $\left(\rho_{n}-\rho_{p}\right) /\left(\rho_{p}+\rho_{n}\right)$ is the isospin asymmetry; $E(\rho, \alpha=0)$ is the binding energy per nucleon in symmetric nuclear matter; and

$$
E_{\text {sym }}(\rho)=\left.\frac{1}{2} \frac{\partial^{2} E(\rho, \alpha)}{\partial \alpha^{2}}\right|_{\alpha=0}
$$

is the nuclear symmetry energy. The absence of odd-order terms in $\alpha$ in Eq. (1) is due to the exchange symmetry between protons and neutrons in nuclear matter when one neglects the Coulomb interaction and assumes the charge symmetry of nuclear forces. The higher-order terms in $\alpha$ are negligible, e.g., the magnitude of the $\alpha^{4}$ term at $\rho_{0}$ is estimated to be less than $1 \mathrm{MeV}$ [60-62]. Neglecting the contribution from higher-order terms in Eq. (1) leads to the well-known empirical parabolic law for the EOS of asymmetric nuclear matter, which has been verified by all many-body theories to date, at least for densities up to moderate values. As a good approximation, the density-dependent symmetry energy $E_{\text {sym }}(\rho)$ can be extracted from $E_{\text {sym }}(\rho) \approx E(\rho, \alpha=1)-E(\rho, \alpha=0)$, i.e., the energy change per nucleon when all protons in the symmetric nuclear matter are converted to neutrons while keeping the total nuclear density fixed. In this sense, the nuclear symmetry energy gives an estimation of the binding energy difference between the pure neutron matter without protons and the symmetric nuclear matter with equal numbers of protons and neutrons. It should be mentioned that the possible presence of the higher-order terms in $\alpha$ at supra-normal densities can significantly modify the proton fraction in $\beta$-equilibrium neutron-star matter and the critical density for the direct Urca process which can lead to faster cooling of neutron stars $[63,64]$.

Around the nuclear matter saturation density $\rho_{0}$, the nuclear symmetry energy $E_{\text {sym }}(\rho)$ can be expanded to second order in density as

$E_{\mathrm{sym}}(\rho)=E_{\mathrm{sym}}\left(\rho_{0}\right)+\frac{L}{3}\left(\frac{\rho-\rho_{0}}{\rho_{0}}\right)+\frac{K_{\mathrm{sym}}}{18}\left(\frac{\rho-\rho_{0}}{\rho_{0}}\right)^{2}$,

where $L$ and $K_{\text {sym }}$ are the slope and curvature parameters of the nuclear symmetry energy at $\rho_{0}$, i.e.,

$$
\begin{aligned}
L & =\left.3 \rho_{0} \frac{\partial E_{\mathrm{sym}}(\rho)}{\partial \rho}\right|_{\rho=\rho_{0}}, \\
K_{\text {sym }} & =\left.9 \rho_{0}^{2} \frac{\partial^{2} E_{\mathrm{sym}}(\rho)}{\partial^{2} \rho}\right|_{\rho=\rho_{0}} .
\end{aligned}
$$


The $L$ and $K_{\text {sym }}$ characterize the density dependence of the nuclear symmetry energy around normal nuclear matter density and thus carry important information on the properties of nuclear symmetry energy at both high and low densities. In particular, the slope parameter $L$ has been found to correlate linearly with the neutron-skin thickness of heavy nuclei and thus can in principle be determined from the measured thickness of the neutron skin of such nuclei [65-72]. Unfortunately, because of the large uncertainties in the experimental measurements, this has not yet been possible so far.

At the nuclear matter saturation density and around $\alpha=0$, the isobaric incompressibility of asymmetric nuclear matter can also be expressed to second order in $\alpha$ as $[73,74]$

$$
K(\alpha) \approx K_{0}+K_{\text {asy }} \alpha^{2},
$$

where $K_{0}$ is the incompressibility of symmetric nuclear matter at the nuclear matter saturation density, and the isospindependent part [75]

$$
K_{\text {asy }} \approx K_{\text {sym }}-6 L
$$

characterizes the density dependence of the nuclear symmetry energy. Information on $K_{\text {asy }}$ can in principle be extracted experimentally by measuring the GMR in neutron-rich nuclei. Earlier attempts based on this method have given, however, widely different values. For example, a value of $K_{\text {asy }}=$ $-320 \pm 180 \mathrm{MeV}$ with a large uncertainty was obtained in Ref. [76] from a systematic study of the GMR in the isotopic chains of $\mathrm{Sn}$ and $\mathrm{Sm}$. In this analysis, the value of $K_{0}$ was found to be $300 \pm 25 \mathrm{MeV}$, which is somewhat larger than the commonly accepted value of $230 \pm 10 \mathrm{MeV}$. In a later study, an even less stringent constraint of $-566 \pm 1350<K_{\text {asy }}<$ $139 \pm 1617 \mathrm{MeV}$ was extracted from the GMR of finite nuclei, depending on the mass region of nuclei and the number of parameters used in parametrizing the incompressibility of finite nuclei [33]. Most recently, a much more stringent constraint of $K_{\text {asy }}=-550 \pm 100 \mathrm{MeV}$ has been obtained in Ref. [59] from measurements of the isotopic dependence of the GMR in even- $A$ Sn isotopes.

Besides studies of nuclear structure, heavy-ion reactions, especially those induced by radioactive beams, also provide a useful means to investigate in terrestrial laboratories the isospin-dependent properties of asymmetric nuclear matter, particularly the density dependence of the nuclear symmetry energy. Indeed, significant progress has recently been made both experimentally and theoretically in extracting the information on the behaviors of nuclear symmetry energy at subsaturation density from the isospin diffusion data in heavy-ion collisions from the National Superconducting Cyclotron Laboratory at Michigan State University (NSCL/MSU) [55-57]. Based on the isospin and momentum-dependent IBUU04 transport model with in-medium $N N$ cross sections, it has been found that the isospin diffusion data are consistent with a density-dependent symmetry energy of $E_{\text {sym }}(\rho) \approx 31.6\left(\rho / \rho_{0}\right)^{\gamma}$ with $\gamma=0.69-1.05$ at subnormal density [56,57], which has led to the extraction of a value of $L=88 \pm 25 \mathrm{MeV}$ for the slope parameter of the nuclear symmetry energy at saturation density and a value of $K_{\text {asy }}=-500 \pm 50 \mathrm{MeV}$ for the isospin-dependent part of the isobaric incompressibility of isospin asymmetric nuclear matter $[56,57,71]$. This has further imposed stringent constraints on both the parameters in the isospin-dependent nuclear effective interactions and the neutron skin thickness of heavy nuclei. Among the 21 sets of Skyrme interactions commonly used in nuclear structure studies, only the four sets $\mathrm{SIV}, \mathrm{SV}, \mathrm{G}_{\sigma}$, and $\mathrm{R}_{\sigma}$ have been found to give symmetry energies that are consistent with the above extracted one. Using these Skyrme interactions, the neutron-skin thickness of heavy nuclei calculated within the Hartree-Fock approach is consistent with available experimental data [71,72] and also that from a relativistic mean-field model based on an accurately calibrated parameter set that reproduces the GMR in ${ }^{90} \mathrm{Zr}$ and ${ }^{208} \mathrm{~Pb}$ as well as the isovector giant dipole resonance of ${ }^{208} \mathrm{~Pb}$ [77]. The extracted symmetry energy further agrees with the symmetry energy $E_{\mathrm{sym}}(\rho)=31.6\left(\rho / \rho_{0}\right)^{0.69}$ recently obtained from the isoscaling analyses of isotope ratios in intermediate energy heavy-ion collisions [58], which gives $L \approx 65 \mathrm{MeV}$ and $K_{\text {asy }} \approx-453 \mathrm{MeV}$. The extracted value of $K_{\text {asy }}=-500 \pm 50 \mathrm{MeV}$ from the isospin diffusion data is also consistent with the value $K_{\text {asy }}=-550 \pm 100 \mathrm{MeV}$ obtained from recently measured isotopic dependence of the GMR in even- $A \mathrm{Sn}$ isotopes [59]. We note that the GMR only allows us to extract the value of $K_{\text {asy }}$ but not that of $L$. These empirically extracted values for $L$ and $K_{\text {asy }}$ represent the best and most stringent phenomenological constraints available so far on the nuclear symmetry energy at subnormal densities. Although the behavior of the symmetry energy at high densities is presently largely undetermined, much of this information is expected to be obtained from future high energy radioactive beam facilities.

\section{B. Nuclear symmetry potential}

The nuclear symmetry potential refers to the isovector part of the nucleon mean-field potential in isospin asymmetric nuclear matter. Besides the nuclear density, the symmetry potential of a nucleon in nuclear matter also depends on the momentum or energy of the nucleon. The nuclear symmetry potential is different from the nuclear symmetry energy, as the latter involves the integration of the isospin-dependent mean-field potential of a nucleon over its momentum. The nuclear symmetry potential is thus a dynamical quantity, while the nuclear symmetry energy is a thermodynamic quantity, and both are essential for understanding many important questions in nuclear physics and astrophysics. Various microscopic and phenomenological models have been used to study the symmetry potential [12,16-31], and the predicted results vary widely as in the case of the nuclear symmetry energy. In particular, whereas most models predict a decreasing symmetry potential with increasing nucleon momentum albeit at different rates, a few nuclear effective interactions used in some models give an opposite behavior.

The nuclear symmetry potential was originally defined in nonrelativistic models. In particular, the nuclear symmetry potential can be evaluated from

$$
U_{\mathrm{sym}}(\rho, \vec{p})=\frac{U_{n}(\rho, \vec{p})-U_{p}(\rho, \vec{p})}{2 \alpha}
$$


where $U_{n}(\rho, \vec{p})$ and $U_{p}(\rho, \vec{p})$ represent, respectively, the neutron and proton single-particle or mean-field potentials. In relativistic models, the nuclear symmetry potential can be similarly defined by using the nonrelativistic reduction of the relativistic single-nucleon potentials. The nuclear symmetry potential in relativistic models therefore depends on the definition of the real part of the nonrelativistic optical potential or the nucleon mean-field potential deduced from the relativistic effective interactions, which are characterized by Lorentz covariant nucleon self-energies. In the relativistic mean-field approximation, these self-energies appear in the single-nucleon Dirac equation

$$
\left[\gamma_{\mu}\left(i \partial^{\mu}-\Sigma_{\tau}^{\mu}\right)-\left(M_{\tau}+\Sigma_{\tau}^{S}\right)\right] \psi_{\tau}=0, \quad \tau=n, p
$$

as the isospin-dependent nucleon vector self-energy $\Sigma_{\tau}^{\mu}$ and scalar self-energy $\Sigma_{\tau}^{S}$. In the Hartree approximation at the static limit, there are no currents in a nucleus or nuclear matter, and the spatial vector components vanish and only the timelike component of the vector self-energy $\Sigma_{\tau}^{0}$ remains. Furthermore, the nucleon self-energy is an energy-independent real and local quantity in the standard RMF model.

There are different methods of deriving the real part of the nonrelativistic optical potential based on the Dirac equation with Lorentz covariant nucleon vector and scalar self-energies. The most popular one is the so-called Schrödinger-equivalent potential (SEP). From the nucleon scalar self-energy $\Sigma_{\tau}^{S}$ and the timelike component of the vector self-energy $\Sigma_{\tau}^{0}$, the SEP is given by [78]

$$
\begin{aligned}
U_{\mathrm{SEP}, \tau} & =\Sigma_{\tau}^{S}+\frac{1}{2 M_{\tau}}\left[\left(\Sigma_{\tau}^{S}\right)^{2}-\left(\Sigma_{\tau}^{0}\right)^{2}\right]+\frac{\Sigma_{\tau}^{0}}{M_{\tau}} E_{\tau} \\
& =\Sigma_{\tau}^{S}+\Sigma_{\tau}^{0}+\frac{1}{2 M_{\tau}}\left[\left(\Sigma_{\tau}^{S}\right)^{2}-\left(\Sigma_{\tau}^{0}\right)^{2}\right]+\frac{\Sigma_{\tau}^{0}}{M_{\tau}} E_{\mathrm{kin}},
\end{aligned}
$$

where $E_{\text {kin }}$ is the kinetic energy of a nucleon, i.e., $E_{\text {kin }}=E_{\tau}-$ $M_{\tau}$ with $E_{\tau}$ being its total energy. Equation (10) shows that $U_{\mathrm{SEP}, \tau}$ increases linearly with the nucleon energy $E_{\tau}$ or kinetic energy $E_{\text {kin }}$ if the nucleon self-energies are independent of energy. We note that by construction, solving the Schrödinger equation with the above SEP gives the same bound-state energy eigenvalues and elastic phase shifts as the solution of the upper component of the Dirac spinor in the Dirac equation with the same nucleon scalar self-energy and timelike component of the vector self-energy [78]. The above SEP thus best represents the real part of the nucleon optical potential in nonrelativistic models $[20,79]$. The corresponding nuclear symmetry potential is given by

$$
U_{\mathrm{sym}}^{\mathrm{SEP}}=\frac{U_{\mathrm{SEP}, n}-U_{\mathrm{SEP}, p}}{2 \alpha},
$$

with $\alpha$ being the isospin asymmetry.

Another popular alternative for deriving the nonrelativistic nucleon optical potential in relativistic models is to take it as the difference between the total energy $E_{\tau}$ of a nucleon with momentum $\vec{p}$ in the nuclear medium and its energy at the same momentum in free space [80], i.e.,

$$
\begin{aligned}
U_{\mathrm{OPT}, \tau} & =E_{\tau}-\sqrt{\mathbf{p}^{2}+M_{\tau}^{2}} \\
& =E_{\tau}-\sqrt{\left(E_{\tau}-\Sigma_{\tau}^{0}\right)^{2}-\Sigma_{\tau}^{S}\left(2 M_{\tau}+\Sigma_{\tau}^{S}\right)} .
\end{aligned}
$$

In obtaining the last step in above equation, the dispersion relation

$$
E_{\tau}=\Sigma_{\tau}^{0}+\sqrt{\mathbf{p}^{2}+\left(M_{\tau}+\Sigma_{\tau}^{S}\right)^{2}}
$$

has been used. This definition for the nucleon optical potential has also been extensively used in microscopic DBHF calculations [81] and transport models for heavy-ion collisions [82]. For energy-independent nucleon self-energies, $U_{\mathrm{OPT}, \tau}$ approaches a constant value of $\Sigma_{\tau}^{0}$ when $|\vec{p}| \rightarrow \infty$, unlike the linear increase of $U_{\mathrm{SEP}, \tau}$ with the nucleon energy. For $|\vec{p}|=0$, we have $U_{\mathrm{OPT}, \tau}=\Sigma_{\tau}^{S}+\Sigma_{\tau}^{0}$ while $U_{\mathrm{SEP}, \tau}=\Sigma_{\tau}^{S}+$ $\Sigma_{\tau}^{0}+\left(\Sigma_{\tau}^{S}+\Sigma_{\tau}^{0}\right)^{2} /\left(2 M_{\tau}\right)$. Therefore, $U_{\mathrm{OPT}, \tau}$ displays a more reasonable high energy behavior than $U_{\mathrm{SEP}, \tau}$. We note that unlike $U_{\mathrm{SEP}, \tau}, U_{\mathrm{OPT}, \tau}$ does not give the same bound-state energy eigenvalues and elastic phase shifts as the solution of the upper component of the Dirac equation. As in the case of $U_{\mathrm{SEP}, \tau}$, the symmetry potential in this approach is defined by

$$
U_{\mathrm{sym}}^{\mathrm{OPT}}=\frac{U_{\mathrm{OPT}, n}-U_{\mathrm{OPT}, p}}{2 \alpha} .
$$

In Ref. [83], another optical potential was introduced based on the second-order Dirac (SOD) equation, and it corresponds to multiplying Eq. (10) by the factor $M_{\tau} / E_{\tau}$, i.e.,

$$
\begin{aligned}
U_{\mathrm{SOD}, \tau} & =\left[\Sigma_{\tau}^{S}+\frac{1}{2 M_{\tau}}\left[\left(\Sigma_{\tau}^{S}\right)^{2}-\left(\Sigma_{\tau}^{0}\right)^{2}\right]+\frac{\Sigma_{\tau}^{0}}{M_{\tau}} E_{\tau}\right] \frac{M_{\tau}}{E_{\tau}} \\
& =\Sigma_{\tau}^{0}+\frac{M_{\tau}}{E_{\tau}} \Sigma_{\tau}^{S}+\frac{1}{2 E_{\tau}}\left[\left(\Sigma_{\tau}^{S}\right)^{2}-\left(\Sigma_{\tau}^{0}\right)^{2}\right] .
\end{aligned}
$$

For energy-independent nucleon self-energies, $U_{\mathrm{SOD}, \tau}$ has the same asymptotical value of $\Sigma_{\tau}^{0}$ as $U_{\mathrm{OPT}, \tau}$ when $|\vec{p}| \rightarrow$ $\infty$. For $|\vec{p}|=0$, we have $U_{\mathrm{SOD}, \tau}=\Sigma_{\tau}^{0}+\frac{M_{\tau}}{\Sigma_{\tau}^{S}+\Sigma_{\tau}^{0}+M_{\tau}} \Sigma_{\tau}^{S}+$ $\frac{1}{2\left(\Sigma_{\tau}^{S}+\Sigma_{\tau}^{0}+M_{\tau}\right)}\left[\left(\Sigma_{\tau}^{S}\right)^{2}-\left(\Sigma_{\tau}^{0}\right)^{2}\right]$. The symmetry potential based on the optical potential of Eq. (15) is given by

$$
U_{\mathrm{sym}}^{\mathrm{SOD}}=\frac{U_{\mathrm{SOD}, n}-U_{\mathrm{SOD}, p}}{2 \alpha} .
$$

The above discussions thus show that the optical potentials defined in Eqs. (12) and (15) have similar high energy behaviors, but they may be very different from that defined in Eq. (10). If we assume that $\Sigma_{\tau}^{S}+\Sigma_{\tau}^{0} \ll M_{\tau}$ and $\left|\Sigma_{\tau}^{S}\right| \approx$ $\left|\Sigma_{\tau}^{0}\right|$, which has been shown to be generally valid in the RMF model even at higher baryon densities, we have, however, $U_{\mathrm{SEP}, \tau} \approx U_{\mathrm{SOD}, \tau} \approx U_{\mathrm{OPT}, \tau}=\Sigma_{\tau}^{S}+\Sigma_{\tau}^{0}$ at low momenta $(|\vec{p}| \approx 0)$, indicating that above three definitions for the optical potential in the RMF model behave similarly at low energies. However, it should be stressed that among the three optical potentials defined above, only $U_{\mathrm{SEP}, \tau}$ is obtained from a welldefined theoretical procedure and is Schrödinger-equivalent, whereas $U_{\mathrm{OPT}, \tau}$ and $U_{\mathrm{SOD}, \tau}$ are used here for heuristic reasons, because they are of practical interest in microscopic DBHF calculations, transport models for heavy-ion collisions, and Dirac phenomenology. 
Empirically, a systematic analysis of a large number of nucleon-nucleus scattering experiments and $(p, n)$ chargeexchange reactions at beam energies up to about $100 \mathrm{MeV}$ has shown that the data can be very well described by the parametrization $U_{\text {sym }}=a-b E_{\text {kin }}$ with $a \approx 22-34 \mathrm{MeV}$ and $b \approx 0.1-0.2$ [84-87]. Although the uncertainties in both parameters $a$ and $b$ are large, the nuclear symmetry potential at nuclear matter saturation density, i.e., the Lane potential $U_{\text {Lane }}$ [88], clearly decreases approximately linearly with increasing beam energy $E_{\text {kin. }}$. This provides a stringent constraint on the low energy behavior of the nuclear symmetry potential at saturation density. As we will see in the following, although the predicted energy dependence of nuclear symmetry potential at low energy from the RMF models does not agree with the empirical Lane potential, it is consistent with results from microscopic DBHF [17], the extended BHF with three-body forces [24], and chiral perturbation theory calculations [89], which give a Lane potential that also stays as a constant or increases slightly with momentum for nucleons with momenta less than about $250-300 \mathrm{MeV} / \mathrm{c}$ or with kinetic energies $E_{\text {kin }}<0$ but decreases with momentum when the momentum is larger than about $250-300 \mathrm{MeV} / c$.

Recently, the high energy behavior of the nuclear symmetry potential has been studied in the relativistic impulse $(t-\rho)$ approximation based on the empirical $N N$ scattering amplitude [90]. The results indicate that the nuclear symmetry potential derived from the Schrödinger-equivalent potential at a fixed density becomes almost constant when the nucleon kinetic energy is greater than about $500 \mathrm{MeV}$, independent of the parameters used in the analysis. It is further shown that for such high energy nucleons, the nuclear symmetry potential is slightly negative at baryon densities below about $\rho=$ $0.22 \mathrm{fm}^{-3}$ and then increases almost linearly to positive values at high densities. These results provide important constraints on the high energy behavior of the nuclear symmetry potential in asymmetric nuclear matter. Furthermore, with the LoveFraney $N N$ scattering amplitude developed by Murdock and Horowitz $[91,92]$, the intermediate energy $\left(100 \leqslant E_{\text {kin }} \leqslant\right.$ $400 \mathrm{MeV}$ ) behavior of the nuclear symmetry potential constructed from the Schrödinger-equivalent potential in isospin asymmetric nuclear matter has also been investigated recently [93]. It shows that the nuclear symmetry potential at fixed baryon density decreases with increasing nucleon energy. In particular, the nuclear symmetry potential at saturation density changes from positive to negative values at nucleon kinetic energy of about $200 \mathrm{MeV}$. Such an energy and density dependence of the nuclear symmetry potential is consistent with those from the isospin- and momentumdependent MDI interaction with $x=0$ (see the Appendix for details on this interaction). These results thus provide an important consistency check for the energy/momentum dependence of the nuclear symmetry potential in asymmetric nuclear matter, particularly the MDI interaction with $x=0$. On the other other, the low energy behavior of the nuclear symmetry potential at densities away from normal nuclear density is presently not known empirically. Experimental determination of both the density and momentum dependence of the nuclear symmetry potential is thus of great interest, and heavy-ion reactions with radioactive beams provides a unique tool to extract this information in terrestrial laboratories.

\section{Nucleon effective mass}

Many different definitions for the nucleon effective mass can be found in the literature [20,79]. In the present work, we mainly focus on the following three effective masses: the Dirac mass $M_{\text {Dirac }}^{*}$ (also denoted as $M^{*}$ in the present work), the Landau mass $M_{\text {Landau }}^{*}$, and the Lorentz mass $M_{\text {Lorentz }}^{*}$. The Dirac mass $M_{\text {Dirac }}^{*}$ is defined through the nucleon scalar self-energy in the Dirac equation, i.e.,

$$
M_{\text {Dirac }, \tau}^{*}=M_{\tau}+\Sigma_{\tau}^{S} .
$$

It is directly related to the spin-orbit potential in finite nuclei and is thus a genuine relativistic quantity without nonrelativistic correspondence. We note that the difference between the nucleon vector and scalar self-energies determines the spin-orbit potential, whereas their sum defines the effective single-nucleon potential and is constrained by the nuclear matter binding energy at saturation density. From the energy spacings between spin-orbit partner states in finite nuclei, the constraint $0.55 M \leqslant M_{\text {Dirac }}^{*} \leqslant 0.6 M$ has been obtained on the value of the Dirac mass $[94,95]$.

The Landau mass $M_{\text {Landau }}^{*}$ is defined as $M_{\text {Landau, } \tau}^{*}=p \frac{d p}{d E_{\tau}}$ in terms of the single-particle density of state $d E_{\tau} / d p$ at energy $E_{\tau}$ and thus characterizes the momentum dependence of the single-particle potential. In the relativistic model, it is given by [94]

$$
M_{\text {Landau }, \tau}^{*}=\left(E_{\tau}-\Sigma_{\tau}^{0}\right)\left(1-\frac{d \Sigma_{\tau}^{0}}{d E_{\tau}}\right)-\left(M_{\tau}+\Sigma_{\tau}^{S}\right) \frac{d \Sigma_{\tau}^{S}}{d E_{\tau}} .
$$

Since $d p / d E_{\tau}$ is in principle measurable, the Landau mass from the relativistic model should have a value comparable to that in the nonrelativistic model. Empirically, based on nonrelativistic effective interactions such as the Skyrme-type interactions, calculations of the ground-state properties and the excitation energies of quadrupole giant resonances have shown that a realistic choice for the nucleon Landau mass is $M_{\text {Landau }}^{*} / M=0.8 \pm 0.1$ [95-98]. The smaller Landau mass than that of the nucleon free mass would lead to a smaller level density at the Fermi energy and much spread single-particle levels in finite nuclei [94].

The Lorentz mass $M_{\text {Lorentz }}^{*}$ characterizes the energy dependence of the Schrödinger-equivalent potential $U_{\mathrm{SEP}, \tau}$ in the relativistic model and is defined as [79]

$$
\begin{aligned}
M_{\text {Lorentz }, \tau}^{*}= & M_{\tau}\left(1-\frac{d U_{\mathrm{SEP}, \tau}}{d E_{\tau}}\right) \\
= & \left(E_{\tau}-\Sigma_{\tau}^{0}\right)\left(1-\frac{d \Sigma_{\tau}^{0}}{d E_{\tau}}\right)-\left(M_{\tau}+\Sigma_{\tau}^{S}\right) \frac{d \Sigma_{\tau}^{S}}{d E_{\tau}} \\
& +M_{\tau}-E_{\tau} \\
= & M_{\text {Landau }, \tau}^{*}+M_{\tau}-E_{\tau} .
\end{aligned}
$$

It has been argued in Ref. [79] that it is the Lorentz mass $M_{\text {Lorentz }}^{*}$ that should be compared with the usual nonrelativistic nucleon effective mass extracted from analyses carried out in 
the framework of nonrelativistic optical and shell models. It can be easily seen that in the nonrelativistic approximation $\left(E_{\tau} \approx M_{\tau}\right.$ ), the Lorentz mass $M_{\text {Lorentz }}^{*}$ reduces to the Landau mass $M_{\text {Landau }}^{*}$.

In relativistic models, the nucleon effective mass has sometimes also been introduced via the energy dependence of the optical potential in Eq. (12) and the second-order Dirac optical potential in Eq. (15), i.e.,

$$
\begin{aligned}
M_{\mathrm{OPT}, \tau}^{*} & =M_{\tau}\left(1-\frac{d U_{\mathrm{OPT}, \tau}}{d E_{\tau}}\right) \\
& =M_{\tau} \frac{\left(E_{\tau}-\Sigma_{\tau}^{0}\right)\left(1-\frac{d \Sigma_{\tau}^{0}}{d E_{\tau}}\right)+\left(M_{\tau}-\Sigma_{\tau}^{S}\right) \frac{d \Sigma_{\tau}^{S}}{d E_{\tau}}}{\sqrt{\left(E_{\tau}-\Sigma_{\tau}^{0}\right)^{2}-\Sigma_{\tau}^{S}\left(2 M_{\tau}+\Sigma_{\tau}^{S}\right)}} \\
& =M_{\tau} \frac{M_{\mathrm{Landau}, \tau}^{*}}{\sqrt{\left(E_{\tau}-\Sigma_{\tau}^{0}\right)^{2}-\Sigma_{\tau}^{S}\left(2 M_{\tau}+\Sigma_{\tau}^{S}\right)}}
\end{aligned}
$$

and

$$
\begin{aligned}
M_{\mathrm{SOD}, \tau}^{*}= & M_{\tau}\left(1-\frac{d U_{\mathrm{SOD}, \tau}}{d E_{\tau}}\right)=M_{\tau}\left[\frac{M_{\text {Landau }, \tau}^{*}}{E_{\tau}}\right. \\
& \left.+\frac{\left(M_{\tau}+\Sigma_{\tau}^{S}\right)^{2}-\left(E_{\tau}-\Sigma_{\tau}^{0}\right)^{2}+E_{\tau}^{2}-M_{\tau}^{2}}{2 E_{\tau}^{2}}\right],
\end{aligned}
$$

respectively.

The isospin splitting of the nucleon effective mass in asymmetric nuclear matter, i.e., the difference between the neutron and proton effective masses is currently not known empirically [99]. Previous theoretical investigations have indicated that most RMF calculations with the isovector $\delta$ meson predict $M_{\text {Dirac }, n}^{*}<M_{\text {Dirac }, p}^{*}$ while in the microscopic DBHF approach, $M_{\text {Dirac }, n}^{*}$ can be larger or smaller than $M_{\text {Dirac, } p}^{*}$ depending on the approximation schemes and methods used for determining the Lorentz and isovector structure of the nucleon self-energy [20]. For the nucleon Lorentz mass, the microscopic DBHF or BHF approach and most nonrelativistic Skyrme-Hartree-Fock calculations predict $M_{\text {Lorentz, } n}^{*}>M_{\text {Lorentz, } p}^{*}$, while most RMF and a few Skyrme-Hartree-Fock calculations give opposite predictions.

\section{RELATIVISTIC MEAN-FIELD MODELS}

For completeness, we briefly introduce in the following the main ingredients in the nonlinear RMF model, the densitydependent RMF model, the nonlinear point-coupling RMF model, and the density-dependent point-coupling RMF model. We neglect the electromagnetic field, because in the present work we are interested in the properties of the infinite nuclear matter. Furthermore, besides the mean-field approximation in which operators of meson fields are replaced by their expectation values (the fields are thus treated as classical c-numbers), we also use the non-sea approximation which neglects the effect due to negative energy states in the Dirac sea.

\section{A. The nonlinear RMF model}

\section{Lagrangian density}

The Lagrangian density in the nonlinear RMF model generally includes the nucleon field $\psi$, the isoscalar-scalar meson field $\sigma$, the isoscalar-vector meson field $\omega$, the isovector-vector meson field $\vec{\rho}$, and the isovector-scalar meson field $\vec{\delta}$, i.e.,

$$
\begin{aligned}
\mathcal{L}_{\mathrm{NL}}= & \bar{\psi}\left[\gamma_{\mu}\left(i \partial^{\mu}-g_{\omega} \omega^{\mu}\right)-\left(M-g_{\sigma} \sigma\right)\right] \psi \\
& +\frac{1}{2}\left(\partial_{\mu} \sigma \partial^{\mu} \sigma-m_{\sigma}^{2} \sigma^{2}\right)-\frac{1}{4} \omega_{\mu \nu} \omega^{\mu \nu}+\frac{1}{2} m_{\omega}^{2} \omega_{\mu} \omega^{\mu} \\
& -\frac{1}{3} b_{\sigma} M\left(g_{\sigma} \sigma\right)^{3}-\frac{1}{4} c_{\sigma}\left(g_{\sigma} \sigma\right)^{4}+\frac{1}{4} c_{\omega}\left(g_{\omega}^{2} \omega_{\mu} \omega^{\mu}\right)^{2} \\
& +\frac{1}{2}\left(\partial_{\mu} \vec{\delta} \cdot \partial^{\mu} \vec{\delta}-m_{\delta}^{2} \vec{\delta}^{2}\right)+\frac{1}{2} m_{\rho}^{2} \vec{\rho}_{\mu} \cdot \vec{\rho}^{\mu}-\frac{1}{4} \vec{\rho}_{\mu \nu} \cdot \vec{\rho}^{\mu \nu} \\
& +\frac{1}{2}\left(g_{\rho}^{2} \vec{\rho}_{\mu} \cdot \vec{\rho}^{\mu}\right)\left(\Lambda_{S} g_{\sigma}^{2} \sigma^{2}+\Lambda_{V} g_{\omega}^{2} \omega_{\mu} \omega^{\mu}\right) \\
& -g_{\rho} \vec{\rho}_{\mu} \cdot \bar{\psi} \gamma^{\mu} \vec{\tau} \psi+g_{\delta} \vec{\delta} \cdot \bar{\psi} \vec{\tau} \psi,
\end{aligned}
$$

where the antisymmetric field tensors $\omega_{\mu \nu}$ and $\vec{\rho}_{\mu \nu}$ are given by $\omega_{\mu \nu} \equiv \partial_{\nu} \omega_{\mu}-\partial_{\mu} \omega_{\nu}$ and $\vec{\rho}_{\mu \nu} \equiv \partial_{\nu} \vec{\rho}_{\mu}-\partial_{\mu} \vec{\rho}_{\nu}$, respectively, and other symbols have their usual meanings. Also, vectors in isospin space are denoted by arrows. This model also contains cross interactions between the isovector meson $\rho$ and isoscalar $\sigma$ and $\omega$ mesons through the cross-coupling constants $\Lambda_{S}$ and $\Lambda_{V}[67,100]$. In addition, we include the isovector-scalar channel ( $\delta$ meson) which is important for the saturation of asymmetric nuclear matter and has also been shown to be an important degree of freedom in describing the properties of asymmetric nuclear matter [101,102]. The above Lagrangian density is quite general and allows us to use most of the presently popular parameter sets in the nonlinear RMF model.

\section{Equation of motion and nucleon self-energies}

With the standard Euler-Lagrange formalism, we can deduce from the Lagrangian density equations of motion for the nucleon and meson fields. The resulting Dirac equation for the nucleon field is

$$
\left[\gamma_{\mu}\left(i \partial^{\mu}-\Sigma_{\tau}^{\mu}\right)-\left(M+\Sigma_{\tau}^{S}\right)\right] \psi=0,
$$

with the following nucleon scalar and vector self-energies:

$$
\begin{aligned}
& \Sigma_{\tau}^{S}=-g_{\sigma} \sigma-g_{\delta} \vec{\delta} \cdot \vec{\tau}, \\
& \Sigma_{\tau}^{\mu}=g_{\omega} \omega^{\mu}+g_{\rho} \vec{\rho}^{\mu} \cdot \vec{\tau} .
\end{aligned}
$$

For the isoscalar meson fields $\sigma$ and $\omega$, they are described by the Klein-Gordon and Proca equations, respectively, i.e.,

$$
\begin{aligned}
\left(\partial_{\mu} \partial^{\mu}+m_{\sigma}^{2}\right) \sigma= & g_{\sigma}\left[\bar{\psi} \psi-b_{\sigma} M\left(g_{\sigma} \sigma\right)^{2}-c_{\sigma}\left(g_{\sigma} \sigma\right)^{3}\right. \\
& \left.+\Lambda_{S}\left(g_{\sigma} \sigma\right) g_{\rho}^{2} \vec{\rho}_{\mu} \cdot \vec{\rho}^{\mu}\right] \\
\partial_{\mu} \omega^{\mu \nu}+m_{\omega}^{2} \omega^{\nu}= & g_{\omega}\left[\bar{\psi} \gamma^{\nu} \psi-c_{\omega} g_{\omega}^{3}\left(\omega_{\mu} \omega^{\mu} \omega^{\nu}\right)\right. \\
& \left.-\Lambda_{V} g_{\rho}^{2} \vec{\rho}_{\mu} \cdot \vec{\rho}^{\mu} g_{\omega} \omega^{\nu}\right] .
\end{aligned}
$$

Analogous equations for the isovector $\delta$ and $\rho$ meson fields are

$$
\begin{aligned}
\left(\partial_{\mu} \partial^{\mu}+m_{\delta}^{2}\right) \vec{\delta}= & g_{\delta} \bar{\psi} \vec{\tau} \psi, \\
\partial_{\mu} \vec{\rho}^{\mu \nu}+m_{\rho}^{2} \vec{\rho}^{v}= & g_{\rho}\left[\bar{\psi} \gamma^{\nu} \vec{\tau} \psi-\Lambda_{S}\left(g_{\rho} \vec{\rho}^{v}\right)\left(g_{\sigma} \sigma\right)^{2}\right. \\
& \left.-\Lambda_{V}\left(g_{\rho} \vec{\rho}^{v}\right) g_{\omega}^{2} \omega_{\mu} \omega^{\mu}\right] .
\end{aligned}
$$


For a static, homogenous infinite nuclear matter, all derivative terms drop out and the expectation values of spacelike components of vector fields vanish (only zero components $\vec{\rho}_{0}$ and $\omega_{0}$ survive) due to translational invariance and rotational symmetry of the nuclear matter. In addition, only the third component of isovector fields $\left(\delta^{(3)}\right.$ and $\left.\rho^{(3)}\right)$ needs to be considered because of the rotational invariance around the third axis in the isospin space. In the mean-field approximation, meson fields are replaced by their expectation values, i.e., $\sigma \rightarrow \bar{\sigma}, \omega_{\mu} \rightarrow \bar{\omega}_{0}, \vec{\delta} \rightarrow \bar{\delta}^{(3)}$, and $\vec{\rho}_{\mu} \rightarrow \bar{\rho}_{0}^{(3)}$, and the meson field equations are reduced to

$$
\begin{aligned}
m_{\sigma}^{2} \bar{\sigma}= & g_{\sigma}\left[\rho_{S}-b_{\sigma} M\left(g_{\sigma} \overline{\bar{\sigma}}\right)^{2}-c_{\sigma}\left(g_{\sigma} \bar{\sigma}\right)^{3}\right. \\
& \left.+\Lambda_{S}\left(g_{\sigma} \bar{\sigma}\right)\left(g_{\rho} \bar{\rho}_{0}^{(3)}\right)^{2}\right], \\
m_{\omega}^{2} \bar{\omega}_{0}= & g_{\omega}\left[\rho_{B}-c_{\omega}\left(g_{\omega} \bar{\omega}_{0}\right)^{3}-\Lambda\left(g_{\omega} \bar{\omega}_{0}\right)\left(g_{\rho} \bar{\rho}_{0}^{(3)}\right)^{2}\right], \\
m_{\delta}^{2} \bar{\delta}^{(3)}= & g_{\delta}\left(\rho_{S, p}-\rho_{S, n}\right) . \\
m_{\rho}^{2} \bar{\rho}_{0}^{(3)}= & g_{\rho}\left[\rho_{B, p}-\rho_{B, n}-\Lambda_{S}\left(g_{\rho} \bar{\rho}_{0}^{(3)}\right)\left(g_{\sigma} \sigma\right)^{2}\right. \\
& \left.-\Lambda_{V}\left(g_{\rho} \bar{\rho}_{0}^{(3)}\right)\left(g_{\omega} \bar{\omega}_{0}\right)^{2}\right] .
\end{aligned}
$$

In the above, the nucleon scalar density $\rho_{S}$ is defined as

$$
\rho_{S}=\langle\bar{\psi} \psi\rangle=\rho_{S, p}+\rho_{S, n},
$$

with the proton $(p)$ and neutron $(n)$ scalar densities given by

$$
\begin{aligned}
\rho_{S, i} & =\frac{2}{(2 \pi)^{3}} \int_{0}^{k_{F}^{i}} d^{3} k \frac{M_{i}^{*}}{\sqrt{\vec{k}^{2}+\left(M_{i}^{*}\right)^{2}}} \\
& =\frac{M_{i}^{*}}{2 \pi^{2}}\left[k_{F}^{i} \tilde{E}_{F}^{i}-\left(M_{i}^{*}\right)^{2} \ln \frac{k_{F}^{i}+\tilde{E}_{F}^{i}}{M_{i}^{*}}\right], \quad i=p, n,
\end{aligned}
$$

where

$$
\tilde{E}_{F}^{i}=\sqrt{\left(k_{F}^{i}\right)^{2}+\left(M_{i}^{*}\right)^{2}},
$$

with $M_{p}^{*}$ and $M_{n}^{*}$ denoting the proton and neutron Dirac masses, respectively, i.e.,

$$
M_{p}^{*}=M-g_{\sigma} \bar{\sigma}-g_{\delta} \bar{\delta}^{(3)}, \quad M_{n}^{*}=M-g_{\sigma} \bar{\sigma}+g_{\delta} \bar{\delta}^{(3)} .
$$

The nucleon scalar and vector self-energies are then given by

$$
\begin{gathered}
\Sigma_{\tau}^{S}=-g_{\sigma} \bar{\sigma}-g_{\delta} \bar{\delta}^{(3)} \tau_{3}, \\
\Sigma_{\tau}^{0}=g_{\omega} \bar{\omega}_{0}+g_{\rho} \bar{\rho}_{0}^{(3)} \tau_{3},
\end{gathered}
$$

with $\tau_{3}=1$ and -1 for protons and neutrons, respectively.

\section{Nuclear matter equation of state}

The set of coupled equations for the nucleon and meson fields can be solved self-consistently using the iteration method, and the properties of the nuclear matter can then be obtained from these fields. From the resulting energymomentum tensor, we can calculate the energy density $\epsilon$ and pressure $P$ of asymmetric nuclear matter, and the results are given by

$$
\begin{aligned}
\epsilon= & \epsilon_{\text {kin }}^{n}+\epsilon_{\text {kin }}^{p} \\
& +\frac{1}{2}\left[m_{\sigma}^{2} \bar{\sigma}^{2}+m_{\omega}^{2} \bar{\omega}_{0}^{2}+m_{\delta}^{2} \bar{\delta}^{(3) 2}+m_{\rho}^{2} \bar{\rho}_{0}^{(3) 2}\right] \\
& +\frac{1}{3} b_{\sigma} M\left(g_{\sigma} \bar{\sigma}\right)^{3}+\frac{1}{4} c_{\sigma}\left(g_{\sigma} \bar{\sigma}\right)^{4}+\frac{3}{4} c_{\omega}\left(g_{\omega} \bar{\omega}_{0}\right)^{4} \\
& +\frac{1}{2}\left(g_{\rho} \bar{\rho}_{0}^{(3)}\right)^{2}\left[\Lambda_{S}\left(g_{\sigma} \bar{\sigma}\right)^{2}+3 \Lambda_{V}\left(g_{\omega} \bar{\omega}_{0}\right)^{2}\right]
\end{aligned}
$$

and

$$
\begin{aligned}
P= & P_{\text {kin }}^{n}+P_{\text {kin }}^{p} \\
& -\frac{1}{2}\left[m_{\sigma}^{2} \bar{\sigma}^{2}-m_{\omega}^{2} \bar{\omega}_{0}^{2}+m_{\delta}^{2} \bar{\delta}^{(3) 2}-m_{\rho}^{2} \bar{\rho}_{0}^{(3) 2}\right] \\
& -\frac{1}{3} b_{\sigma} M\left(g_{\sigma} \bar{\sigma}\right)^{3}-\frac{1}{4} c_{\sigma}\left(g_{\sigma} \bar{\sigma}\right)^{4}+\frac{1}{4} c_{\omega}\left(g_{\omega} \bar{\omega}_{0}\right)^{4} \\
& +\frac{1}{2}\left(g_{\rho} \bar{\rho}_{0}^{(3)}\right)^{2}\left[\Lambda_{S}\left(g_{\sigma} \bar{\sigma}\right)^{2}+\Lambda_{V}\left(g_{\omega} \bar{\omega}_{0}\right)^{2}\right] .
\end{aligned}
$$

In the above, $\epsilon_{\mathrm{kin}}^{i}$ and $P_{\mathrm{kin}}^{i}$ are, respectively, the kinetic contributions to the energy densities and pressure of protons and neutrons in nuclear matter, and they are given by

$$
\begin{aligned}
\epsilon_{\mathrm{kin}}^{i} & =\frac{2}{(2 \pi)^{3}} \int_{0}^{k_{F}^{i}} d^{3} k \sqrt{\vec{k}^{2}+\left(M_{i}^{*}\right)^{2}} \\
& =\frac{1}{4}\left[3 \tilde{E}_{F}^{i} \rho_{B, i}+M_{i}^{*} \rho_{S, i}\right], \quad i=p, n,
\end{aligned}
$$

and

$$
\begin{aligned}
P_{\mathrm{kin}}^{i} & =\frac{2}{3(2 \pi)^{3}} \int_{0}^{k_{F}^{i}} d^{3} k \frac{\vec{k}^{2}}{\sqrt{\vec{k}^{2}+\left(M_{i}^{*}\right)^{2}}} \\
& =\frac{1}{4}\left[\tilde{E}_{F}^{i} \rho_{B, i}-M_{i}^{*} \rho_{S, i}\right], \quad i=p, n .
\end{aligned}
$$

The binding energy per nucleon can be obtained from the energy density via

$$
E=\frac{\epsilon}{\rho_{B}}-M,
$$

while the symmetry energy is given by

$$
\begin{aligned}
E_{\mathrm{sym}}\left(\rho_{B}\right)= & \frac{k_{F}^{2}}{6 \tilde{E}_{F}}+\frac{1}{2}\left(\frac{g_{\rho}}{m_{\rho}^{*}}\right)^{2} \rho_{B}-\frac{1}{2}\left(\frac{g_{\delta}}{m_{\delta}}\right)^{2} \\
& \times \frac{M^{* 2} \rho_{B}}{\tilde{E}_{F}^{2}\left[1+\left(\frac{g_{\delta}}{m_{\delta}}\right)^{2} A\left(k_{F}, M^{*}\right)\right]},
\end{aligned}
$$

with the effective $\rho$-meson mass given by [67]

$$
m_{\rho}^{* 2}=m_{\rho}^{2}+g_{\rho}^{2}\left[\Lambda_{S}\left(g_{\sigma} \bar{\sigma}\right)^{2}+\Lambda_{V}\left(g_{\omega} \bar{\omega}_{0}\right)^{2}\right]
$$

and

$$
\begin{aligned}
A\left(k_{F}, M^{*}\right) & =\frac{4}{(2 \pi)^{3}} \int_{0}^{k_{F}} d^{3} k \frac{\vec{k}^{2}}{\left(\vec{k}^{2}+\left(M^{*}\right)^{2}\right)^{3 / 2}} \\
& =3\left(\frac{\rho_{S}}{M^{*}}-\frac{\rho_{B}}{\tilde{E}_{F}}\right),
\end{aligned}
$$

where $\tilde{E}_{F}=\sqrt{k_{F}^{2}+M^{* 2}}$ and $M^{*}$ is the nucleon Dirac mass in symmetric nuclear matter. 


\section{B. The density-dependent RMF model}

\section{Lagrangian density}

In the density-dependent RMF model, instead of introducing terms involving self-interactions of the scalar meson field and cross-interactions of meson fields as in the nonlinear RMF model, the coupling constants are density dependent. The Lagrangian density in this model is generally written as

$$
\begin{aligned}
\mathcal{L}_{\mathrm{DD}}= & \bar{\psi}\left[\gamma_{\mu}\left(i \partial^{\mu}-\Gamma_{\omega} \omega^{\mu}-\Gamma_{\rho} \vec{\rho}^{\mu} \cdot \vec{\tau}\right)\right. \\
& \left.-\left(M-\Gamma_{\sigma} \sigma-\Gamma_{\delta} \vec{\delta} \cdot \vec{\tau}\right)\right] \psi+\frac{1}{2}\left(\partial_{\mu} \sigma \partial^{\mu} \sigma-m_{s}^{2} \sigma^{2}\right) \\
& +\frac{1}{2}\left(\partial_{\mu} \vec{\delta} \cdot \partial^{\mu} \vec{\delta}-m_{\delta}^{2} \vec{\delta}^{2}\right)-\frac{1}{4} \omega_{\mu \nu} \omega^{\mu \nu}+\frac{1}{2} m_{\omega}^{2} \omega_{\mu} \omega^{\mu} \\
& -\frac{1}{4} \vec{\rho}_{\mu \nu} \cdot \vec{\rho}^{\mu \nu}+\frac{1}{2} m_{\rho}^{2} \vec{\rho}_{\mu} \cdot \vec{\rho}^{\mu} .
\end{aligned}
$$

The symbols used in this equation have their usual meanings, as in Eq. (22), but the coupling constants $\Gamma_{\sigma}, \Gamma_{\omega}, \Gamma_{\delta}$, and $\Gamma_{\rho}$ now depend on the (baryon) density and are usually parametrized as

$$
\Gamma_{i}(\rho)=\Gamma_{i}\left(\rho_{\mathrm{sat}}\right) h_{i}(x), \quad x=\rho / \rho_{\mathrm{sat}},
$$

with

$$
h_{i}(x)=a_{i} \frac{1+b_{i}\left(x+d_{i}\right)^{2}}{1+c_{i}\left(x+e_{i}\right)^{2}}, \quad i=\sigma, \omega, \delta, \rho,
$$

and $\rho_{\text {sat }}$ being the saturation density of symmetric nuclear matter. In some parameter sets,

$$
h_{\rho}(x)=\exp \left[-a_{\rho}(x-1)\right]
$$

is used for the $\rho$ meson.

\section{Equation of motion and nucleon self-energies}

Since the coupling constants in the density-dependent RMF model depend on the baryon fields $\bar{\psi}$ and $\psi$ through the density, additional terms besides the usual ones in the nonlinear RMF model appear in the field equations of motion when the partial derivatives of $\mathcal{L}_{\mathrm{DD}}$ are carried out with respect to the fields $\bar{\psi}$ and $\psi$ in the Euler-Lagrange equations. The resulting Dirac equation for the nucleon field now reads

$$
\left[\gamma_{\mu}\left(i \partial^{\mu}-\Sigma_{\tau}^{\mu}\right)-\left(M+\Sigma_{\tau}^{S}\right)\right] \psi=0,
$$

with the following nucleon scalar and vector self-energies:

$$
\begin{aligned}
& \Sigma_{\tau}^{S}=-\Gamma_{\sigma} \sigma-\Gamma_{\delta} \vec{\delta} \cdot \vec{\tau}, \\
& \Sigma_{\tau}^{\mu}=\Gamma_{\omega} \omega^{\mu}+\Gamma_{\rho} \vec{\rho}^{\mu} \cdot \vec{\tau}+\Sigma^{\mu(R)} .
\end{aligned}
$$

The new term $\Sigma^{\mu(R)}$ in the vector self-energy, which is called the rearrangement self-energy $[44,45]$, is given by

$$
\begin{aligned}
\Sigma^{\mu(R)}= & \frac{j^{\mu}}{\rho}\left(\frac{\partial \Gamma_{\omega}}{\partial \rho} \bar{\psi} \gamma_{\nu} \psi \omega^{\nu}+\frac{\partial \Gamma_{\rho}}{\partial \rho} \bar{\psi} \vec{\tau} \gamma^{\nu} \psi \cdot \vec{\rho}_{\nu}\right. \\
& \left.-\frac{\partial \Gamma_{\sigma}}{\partial \rho} \bar{\psi} \psi \sigma-\frac{\partial \Gamma_{\delta}}{\partial \rho} \bar{\psi} \vec{\tau} \psi \vec{\delta}\right),
\end{aligned}
$$

with $j^{\mu}=\bar{\psi} \gamma^{\mu} \psi$. The rearrangement self-energy plays an essential role in the application of the theory since it guarantees both the thermodynamic consistency and the energymomentum conservation $[44,45]$.
For the meson fields, the equations of motion are

$$
\begin{aligned}
\left(\partial_{\mu} \partial^{\mu}+m_{\sigma}^{2}\right) \sigma & =\Gamma_{\sigma} \bar{\psi} \psi, \\
\partial_{\nu} \omega^{\mu \nu}+m_{\omega}^{2} \omega^{\mu} & =\Gamma_{\omega} \bar{\psi} \gamma^{\mu} \psi, \\
\left(\partial_{\mu} \partial^{\mu}+m_{\delta}^{2}\right) \vec{\delta} & =\Gamma_{\delta} \bar{\psi} \vec{\tau} \psi, \\
\partial_{\nu} \vec{\rho}^{\mu \nu}+m_{\rho}^{2} \vec{\rho}^{\mu} & =\Gamma_{\rho} \bar{\psi} \vec{\tau} \gamma^{\mu} \psi .
\end{aligned}
$$

In the static case for an infinite nuclear matter, the meson equations of motion become

$$
\begin{aligned}
m_{\sigma}^{2} \bar{\sigma} & =\Gamma_{\sigma} \rho_{S}, \\
m_{\omega}^{2} \bar{\omega}_{0} & =\Gamma_{\omega} \rho_{B}, \\
m_{\rho}^{2} \bar{\rho}_{0}^{(3)} & =\Gamma_{\rho}\left(\rho_{p}-\rho_{n}\right), \\
m_{\delta}^{2} \bar{\delta}^{(3)} & =\Gamma_{\delta}\left(\rho_{S, p}-\rho_{S, n}\right),
\end{aligned}
$$

so the nucleon scalar and vector self-energies are

$$
\begin{aligned}
& \Sigma_{\tau}^{S}=-\Gamma_{\sigma} \bar{\sigma}-\Gamma_{\delta} \bar{\delta}^{(3)} \tau_{3}, \\
& \Sigma_{\tau}^{0}=\Gamma_{\omega} \bar{\omega}_{0}+\Gamma_{\rho} \bar{\rho}_{0}^{(3)} \tau_{3}+\Sigma^{0(R)},
\end{aligned}
$$

with

$$
\begin{aligned}
\Sigma^{0(R)}= & \frac{\partial \Gamma_{\omega}}{\partial \rho} \bar{\omega}_{0} \rho_{B}+\frac{\partial \Gamma_{\rho}}{\partial \rho} \bar{\rho}_{0}^{(3)}\left(\rho_{p}-\rho_{n}\right) \\
& -\frac{\partial \Gamma_{\sigma}}{\partial \rho} \bar{\sigma} \rho_{S}-\frac{\partial \Gamma_{\delta}}{\partial \rho} \bar{\delta}^{(3)}\left(\rho_{S, p}-\rho_{S, n}\right) .
\end{aligned}
$$

\section{Nuclear matter equation of state}

From the energy-momentum tensor in the densitydependent RMF model, the energy density and pressure of nuclear matter can be derived, and they are given by

$$
\epsilon=\epsilon_{\mathrm{kin}}^{n}+\epsilon_{\mathrm{kin}}^{p}+\frac{1}{2}\left[m_{\sigma}^{2} \bar{\sigma}^{2}+m_{\omega}^{2} \bar{\omega}_{0}^{2}+m_{\delta}^{2} \bar{\delta}^{(3) 2}+m_{\rho}^{2} \bar{\rho}_{0}^{(3) 2}\right]
$$

and

$$
\begin{aligned}
P= & P_{\mathrm{kin}}^{n}+P_{\mathrm{kin}}^{p}+\rho_{B} \Sigma^{0(R)} \\
& -\frac{1}{2}\left[m_{\sigma}^{2} \bar{\sigma}^{2}-m_{\omega}^{2} \bar{\omega}_{0}^{2}+m_{\delta}^{2} \bar{\delta}^{(3) 2}-m_{\rho}^{2} \bar{\rho}_{0}^{(3) 2}\right] .
\end{aligned}
$$

It is seen that the rearrangement self-energy does not affect the energy density but contributes explicitly to the pressure. Furthermore, the symmetry energy can be written as

$$
\begin{aligned}
E_{\mathrm{sym}}\left(\rho_{B}\right)= & \frac{k_{F}^{2}}{6 \tilde{E}_{F}}+\frac{1}{2}\left(\frac{\Gamma_{\rho}}{m_{\rho}}\right)^{2} \rho_{B}-\frac{1}{2}\left(\frac{\Gamma_{\delta}}{m_{\delta}}\right)^{2} \\
& \times \frac{M^{* 2} \rho_{B}}{\tilde{E}_{F}^{2}\left[1+\left(\frac{\Gamma_{\delta}}{m_{\delta}}\right)^{2} A\left(k_{F}, M^{*}\right)\right]},
\end{aligned}
$$

with notations similarly defined as in the nonlinear RMF model.

\section{The nonlinear point-coupling RMF model}

\section{Lagrangian density}

The point-coupling model is defined by a Lagrangian density that consists of only nucleon fields. In the present study, 
we use the Lagrangian density of the nonlinear point-coupling model of Refs. [49,50], i.e.,

$$
\mathcal{L}_{\mathrm{NLPC}}=\mathcal{L}^{\text {free }}+\mathcal{L}^{4 \mathrm{f}}+\mathcal{L}^{\text {hot }}+\mathcal{L}^{\text {der }},
$$

with

$$
\begin{aligned}
\mathcal{L}^{\text {free }}= & \bar{\psi}\left(\mathrm{i} \gamma_{\mu} \partial^{\mu}-M\right) \psi, \\
\mathcal{L}^{4 \mathrm{f}}= & -\frac{1}{2} \alpha_{S}(\bar{\psi} \psi)(\bar{\psi} \psi)-\frac{1}{2} \alpha_{V}\left(\bar{\psi} \gamma_{\mu} \psi\right)\left(\bar{\psi} \gamma^{\mu} \psi\right) \\
& -\frac{1}{2} \alpha_{T S}(\bar{\psi} \vec{\tau} \psi) \cdot(\bar{\psi} \vec{\tau} \psi)-\frac{1}{2} \alpha_{T V}\left(\bar{\psi} \vec{\tau} \gamma_{\mu} \psi\right) \\
& \cdot\left(\bar{\psi} \vec{\tau} \gamma^{\mu} \psi\right) \\
\mathcal{L}^{\text {hot }}= & -\frac{1}{3} \beta_{S}(\bar{\psi} \psi)^{3}-\frac{1}{4} \gamma_{S}(\bar{\psi} \psi)^{4}-\frac{1}{4} \gamma_{V}\left[\left(\bar{\psi} \gamma_{\mu} \psi\right)\left(\bar{\psi} \gamma^{\mu} \psi\right)\right]^{2} \\
& -\frac{1}{4} \gamma_{T V}\left[\left(\bar{\psi} \vec{\tau} \gamma_{\mu} \psi\right) \cdot\left(\bar{\psi} \vec{\tau} \gamma^{\mu} \psi\right)\right]^{2}, \\
\mathcal{L}^{\text {der }}= & -\frac{1}{2} \delta_{S}\left(\partial_{\nu} \bar{\psi} \psi\right)\left(\partial^{v} \bar{\psi} \psi\right)-\frac{1}{2} \delta_{V}\left(\partial_{\nu} \bar{\psi} \gamma_{\mu} \psi\right)\left(\partial^{v} \bar{\psi} \gamma^{\mu} \psi\right) \\
& -\frac{1}{2} \delta_{T S}\left(\partial_{\nu} \bar{\psi} \vec{\tau} \psi\right) \cdot\left(\partial^{\nu} \bar{\psi} \vec{\tau} \psi\right)-\frac{1}{2} \delta_{T V}\left(\partial_{\nu} \bar{\psi} \vec{\tau} \gamma_{\mu} \psi\right) \\
& \cdot\left(\partial^{\nu} \bar{\psi} \vec{\tau} \gamma^{\mu} \psi\right) .
\end{aligned}
$$

In the above, $\mathcal{L}^{\text {free }}$ is the kinetic term of nucleons and $\mathcal{L}^{4 \mathrm{f}}$ describes the four-fermion interactions, while $\mathcal{L}^{\text {hot }}$ and $\mathcal{L}^{\text {der }}$ contain, respectively, higher-order terms involving more than four fermions and derivatives in the nucleon field. For the 12 coupling constants in the Lagrangian density, $\alpha_{S}, \alpha_{V}, \alpha_{T S}, \alpha_{T V}, \beta_{S}, \gamma_{S}, \gamma_{V}, \gamma_{T V}, \delta_{S}, \delta_{V}, \delta_{T S}$, and $\delta_{T V}$, the subscripts denote the tensor structure of a coupling with $S, V$, and $T$ standing for scalar, vector, and isovector, respectively. The symbols $\alpha_{i}, \delta_{i}, \beta_{i}$, and $\gamma_{i}$ refer, respectively, to fourfermion or second-order terms, derivative couplings, and thirdand fourth-order terms $[49,50]$.

\section{Equation of motion and nucleon self-energies}

From the variation of the Lagrangian density Eq. (69) with respect to $\bar{\psi}$, we obtain the following Dirac equation for the nucleon field:

$$
\left[\gamma_{\mu}\left(i \partial^{\mu}-\Sigma^{\mu}\right)-\left(M+\Sigma^{S}\right)\right] \psi=0,
$$

where the nucleon scalar and vector self-energies are, respectively,

$$
\begin{aligned}
\Sigma^{S} & =V_{S}+\vec{V}_{T S} \cdot \vec{\tau}, \\
\Sigma^{\mu} & =V^{\mu}+\vec{V}_{T}^{\mu} \cdot \vec{\tau},
\end{aligned}
$$

with

$$
\begin{aligned}
V_{S}= & \alpha_{S}(\bar{\psi} \psi)+\beta_{S}(\bar{\psi} \psi)^{2}+\gamma_{S}(\bar{\psi} \psi)^{3}-\delta_{S} \square(\bar{\psi} \psi) \\
\vec{V}_{T S}= & \alpha_{T S}(\bar{\psi} \vec{\tau} \psi)-\delta_{T S} \square(\bar{\psi} \vec{\tau} \psi), \\
V^{\mu}= & \alpha_{V}\left(\bar{\psi} \gamma^{\mu} \psi\right)+\gamma_{V}\left(\bar{\psi} \gamma^{\mu} \psi\right)\left(\bar{\psi} \gamma_{\mu} \psi\right)\left(\bar{\psi} \gamma^{\mu} \psi\right) \\
& -\delta_{V} \square\left(\bar{\psi} \gamma^{\mu} \psi\right), \\
\vec{V}_{T}^{\mu}= & \alpha_{T V}\left(\bar{\psi} \vec{\tau} \gamma^{\mu} \psi\right)+\gamma_{T V}\left(\bar{\psi} \vec{\tau} \gamma^{\mu} \psi\right) \cdot\left(\bar{\psi} \vec{\tau} \gamma_{\mu} \psi\right)\left(\bar{\psi} \vec{\tau} \gamma^{\mu} \psi\right) \\
& -\delta_{T V} \square\left(\bar{\psi} \vec{\tau} \gamma^{\mu} \psi\right) .
\end{aligned}
$$

In the above, $\square=\partial^{2} /\left(c^{2} \partial t^{2}-\triangle\right)$ denotes the fourdimensional D'Alembertian. In the translationally invariant infinite nuclear matter, all terms involving derivative couplings drop out, and the spatial components of the four-currents also vanish. In terms of the baryon density $\rho_{B}$ and scalar density $\rho_{S}$ as well as the isospin baryon density $\rho_{3}=\rho_{p}-\rho_{n}$ and the isospin scalar density $\rho_{S 3}=\rho_{S, p}-\rho_{S, n}$, the nucleon scalar and vector self-energies in asymmetric nuclear matter can be rewritten as

$$
\begin{aligned}
& \Sigma_{\tau}^{S}=\alpha_{S} \rho_{S}+\beta_{S} \rho_{S}^{2}+\gamma_{S} \rho_{S}^{3}+\alpha_{T S} \rho_{S 3} \tau_{3}, \\
& \Sigma_{\tau}^{0}=\alpha_{V} \rho_{B}+\gamma_{V} \rho_{B}^{3}+\alpha_{T V} \rho_{3} \tau_{3}+\gamma_{T V} \rho_{3}^{3} \tau_{3} .
\end{aligned}
$$

\section{Nuclear matter equation of state}

The energy density $\epsilon$ and the pressure $P$ derived from the energy-momentum tensor in the nonlinear point-coupling RMF model are given by

$$
\begin{aligned}
\epsilon= & \epsilon_{\text {kin }}^{n}+\epsilon_{\text {kin }}^{p}-\frac{1}{2} \alpha_{S} \rho_{S}^{2}-\frac{1}{2} \alpha_{T S} \rho_{S 3}^{2}+\frac{1}{2} \alpha_{V} \rho^{2}+\frac{1}{2} \alpha_{T V} \rho_{3}^{2} \\
& -\frac{1}{3} \beta_{S} \rho_{S}^{3}-\frac{3}{4} \gamma_{S} \rho_{S}^{4}+\frac{1}{4} \gamma_{V} \rho^{4}+\frac{1}{4} \gamma_{T V} \rho_{3}^{4}, \\
P= & \tilde{E}_{F}^{p} \rho_{p}+\tilde{E}_{F}^{n} \rho_{n}-\epsilon_{\text {kin }}^{p}-\epsilon_{\text {kin }}^{n}+\frac{1}{2} \alpha_{S} \rho_{s}^{2}+\frac{1}{2} \alpha_{T S} \rho_{s 3}^{2} \\
& +\frac{1}{2} \alpha_{V} \rho^{2}+\frac{1}{2} \alpha_{T V} \rho_{3}^{2}+\frac{2}{3} \beta_{S} \rho_{S}^{3}+\frac{3}{4} \gamma_{S} \rho_{S}^{4}+\frac{3}{4} \gamma_{V} \rho^{4} \\
& +\frac{3}{4} \gamma_{T V} \rho_{3}^{4},
\end{aligned}
$$

where $\tilde{E}_{F}^{p}$ and $\tilde{E}_{F}^{n}$ are defined as in Eq. (36) with the nucleon Dirac masses

$$
\begin{aligned}
& M_{p}^{*}=\alpha_{S} \rho_{S}+\beta_{S} \rho_{S}^{2}+\gamma_{S} \rho_{S}^{3}+\alpha_{T S} \rho_{S 3}, \\
& M_{n}^{*}=\alpha_{S} \rho_{S}+\beta_{S} \rho_{S}^{2}+\gamma_{S} \rho_{S}^{3}-\alpha_{T S} \rho_{S 3} .
\end{aligned}
$$

Furthermore, the symmetry energy in this model can be expressed as

$$
\begin{aligned}
E_{\mathrm{sym}}\left(\rho_{B}\right)= & \frac{k_{F}^{2}}{6 \tilde{E}_{F}}+\frac{1}{2} \alpha_{T V} \rho_{B}+\frac{1}{2} \alpha_{T S} \\
& \times \frac{M^{* 2} \rho_{B}}{\tilde{E}_{F}^{2}\left[1-\alpha_{T S} A\left(k_{F}, M^{*}\right)\right]},
\end{aligned}
$$

with notations again similarly defined as in the nonlinear RMF model.

\section{The density-dependent point-coupling RMF model}

\section{Lagrangian density}

For the density-dependent point-coupling RMF model, we use the Lagrangian density of Refs. [53,54], i.e.,

$$
\mathcal{L}_{\text {DDPC }}=\mathcal{L}_{\text {free }}+\mathcal{L}_{4 \mathrm{f}}+\mathcal{L}_{\text {der }},
$$

with

$$
\begin{aligned}
\mathcal{L}_{\text {free }}= & \bar{\psi}\left(i \gamma_{\mu} \partial^{\mu}-M\right) \psi \\
\mathcal{L}_{4 \mathrm{f}}= & -\frac{1}{2} G_{S}(\hat{\rho})(\bar{\psi} \psi)(\bar{\psi} \psi)-\frac{1}{2} G_{V}(\hat{\rho})\left(\bar{\psi} \gamma_{\mu} \psi\right)\left(\bar{\psi} \gamma^{\mu} \psi\right) \\
& -\frac{1}{2} G_{T S}(\hat{\rho})(\bar{\psi} \vec{\tau} \psi) \cdot(\bar{\psi} \vec{\tau} \psi)-\frac{1}{2} G_{T V}(\hat{\rho})\left(\bar{\psi} \vec{\tau} \gamma_{\mu} \psi\right) \\
& \cdot\left(\bar{\psi} \vec{\tau} \gamma^{\mu} \psi\right), \\
\mathcal{L}_{\text {der }}= & -\frac{1}{2} D_{S}(\hat{\rho})\left(\partial_{\nu} \bar{\psi} \psi\right)\left(\partial^{\nu} \bar{\psi} \psi\right) .
\end{aligned}
$$

In the above, $\mathcal{L}^{\text {free }}$ is the kinetic term of the nucleons, $\mathcal{L}^{4 \mathrm{f}}$ is a four-fermion interaction, and $\mathcal{L}^{\text {der }}$ represents derivatives in the nucleon scalar densities. Unlike in the nonlinear pointcoupling RMF model, the density-dependent point-coupling RMF model used here includes only second-order interaction 
terms with density-dependent couplings $G_{i}(\hat{\rho})$ and $D_{i}(\hat{\rho})$ that are determined from finite-density QCD sum rules and inmedium chiral perturbation theory $[53,54]$.

\section{Equation of motion and nucleon self-energies}

Variation of the Lagrangian density Eq. (88) with respect to $\bar{\psi}$ leads to the single-nucleon Dirac equation

$$
\left[\gamma_{\mu}\left(i \partial^{\mu}-\Sigma^{\mu}\right)-\left(M+\Sigma^{S}\right)\right] \psi=0,
$$

with the nucleon scalar and vector self-energies given, respectively, by

$$
\begin{aligned}
& \Sigma^{S}=V_{S}+\vec{V}_{T S} \cdot \vec{\tau}+\Sigma_{r S}, \\
& \Sigma^{\mu}=V^{\mu}+\vec{V}_{T}^{\mu} \cdot \vec{\tau}+\Sigma_{r}^{\mu},
\end{aligned}
$$

where

$$
\begin{aligned}
V_{S} & =G_{S}(\bar{\psi} \psi)-D_{S} \square(\bar{\psi} \psi), \\
\vec{V}_{T S} & =G_{T S}(\bar{\psi} \vec{\tau} \psi), \\
V^{\mu} & =G_{V}\left(\bar{\psi} \gamma^{\mu} \psi\right), \\
\vec{V}_{T}^{\mu} & =G_{T V}\left(\bar{\psi} \vec{\tau} \gamma^{\mu} \psi\right), \\
\Sigma_{r S} & =-\frac{\partial D_{S}}{\partial \hat{\rho}}\left(\partial_{\nu} j^{\mu}\right) u_{\mu}\left(\partial^{\nu}(\bar{\psi} \psi)\right),
\end{aligned}
$$

and

$$
\begin{aligned}
\Sigma_{r}^{\mu}= & \frac{u^{\mu}}{2}\left(\frac{\partial G_{S}}{\partial \hat{\rho}}(\bar{\psi} \psi)(\bar{\psi} \psi)+\frac{\partial G_{T S}}{\partial \hat{\rho}}(\bar{\psi} \vec{\tau} \psi) \cdot(\bar{\psi} \vec{\tau} \psi)\right. \\
& +\frac{\partial G_{V}}{\partial \hat{\rho}}\left(\bar{\psi} \gamma^{\mu} \psi\right)\left(\bar{\psi} \gamma_{\mu} \psi\right)+\frac{\partial G_{T V}}{\partial \hat{\rho}}\left(\bar{\psi} \vec{\tau} \gamma^{\mu} \psi\right) \\
& \left.\cdot\left(\bar{\psi} \vec{\tau} \gamma_{\mu} \psi\right)+\frac{\partial D_{S}}{\partial \hat{\rho}}\left(\partial^{v}(\bar{\psi} \psi)\right)\left(\partial_{\nu}(\bar{\psi} \psi)\right)\right) \cdot
\end{aligned}
$$

In the above, we have $\hat{\rho} u^{\mu}=\bar{\psi} \gamma^{\mu} \psi$, where the four-velocity $u^{\mu}$ is defined as $\left(1-\mathbf{v}^{2}\right)^{-1 / 2}(1, \mathbf{v})$ with $\mathbf{v}$ being the threevelocity vector, and $\Sigma_{r S}$ and $\Sigma_{r}^{\mu}$ represent the rearrangement contributions resulting from the variation of the vertex functionals with respect to the nucleon fields in the density operator $\hat{\rho}$. The latter coincides with the baryon density in the nuclear matter rest frame.

In the translationally invariant infinite asymmetric nuclear matter, the nucleon scalar and vector self-energies become

$$
\begin{aligned}
& \Sigma_{\tau}^{S}=G_{S} \rho_{S}+G_{T S} \rho_{S 3} \tau_{3} \\
& \Sigma_{\tau}^{0}=G_{V} \rho_{B}+G_{T V} \rho_{3} \tau_{3}+\Sigma^{0(R)},
\end{aligned}
$$

with the rearrangement contribution to the self-energy

$$
\Sigma^{0(R)}=\frac{1}{2}\left[\frac{\partial G_{S}}{\partial \rho} \rho_{S}^{2}+\frac{\partial G_{T S}}{\partial \rho} \rho_{S 3}^{2}+\frac{\partial G_{V}}{\partial \rho} \rho^{2}+\frac{\partial G_{T V}}{\partial \rho} \rho_{3}^{2}\right] .
$$

\section{Nuclear matter equation of state}

For asymmetric nuclear matter, the energy density $\epsilon$ and the pressure $P$ derived from the energy-momentum tensor in the density-dependent point-coupling RMF model are

$$
\begin{aligned}
\epsilon= & \epsilon_{\mathrm{kin}}^{n}+\epsilon_{\mathrm{kin}}^{p}-\frac{1}{2} G_{S} \rho_{S}^{2}-\frac{1}{2} G_{T S} \rho_{S 3}^{2} \\
& +\frac{1}{2} G_{V} \rho^{2}+\frac{1}{2} G_{T V} \rho_{3}^{2},
\end{aligned}
$$

and

$$
\begin{aligned}
P= & \tilde{E}_{F}^{p} \rho_{p}+\tilde{E}_{F}^{n} \rho_{n}-\epsilon_{\mathrm{kin}}^{p}-\epsilon_{\mathrm{kin}}^{n}+\frac{1}{2} G_{V} \rho^{2}+\frac{1}{2} G_{T V} \rho_{3}^{2} \\
& +\frac{1}{2} G_{S} \rho_{S}^{2}+\frac{1}{2} G_{T S} \rho_{S 3}^{2}+\frac{1}{2} \frac{\partial G_{S}}{\partial \rho} \rho_{S}^{2} \rho+\frac{1}{2} \frac{\partial G_{V}}{\partial \rho} \rho^{3} \\
& +\frac{1}{2} \frac{\partial G_{T V}}{\partial \rho} \rho_{3}^{2} \rho+\frac{1}{2} \frac{\partial G_{T S}}{\partial \rho} \rho_{S 3}^{2} \rho
\end{aligned}
$$

where $\tilde{E}_{F}^{p}$ and $\tilde{E}_{F}^{n}$ are defined as in Eq. (36) with the effective nucleon masses

$$
\begin{aligned}
& M_{p}^{*}=M+G_{S} \rho_{S}+G_{T S} \rho_{S 3}, \\
& M_{n}^{*}=M+G_{S} \rho_{S}-G_{T S} \rho_{S 3} .
\end{aligned}
$$

As in the density-dependent RMF model, rearrangement contributions appear explicitly in the expression for the pressure. Finally, the symmetry energy can be written as

$$
\begin{aligned}
E_{\mathrm{sym}}\left(\rho_{B}\right)= & \frac{k_{F}^{2}}{6 \tilde{E}_{F}}+\frac{1}{2} G_{T V} \rho_{B} \\
& +\frac{1}{2} G_{T S} \frac{M^{* 2} \rho_{B}}{\tilde{E}_{F}^{2}\left[1-G_{T S} A\left(k_{F}, M^{*}\right)\right]},
\end{aligned}
$$

with similar notation as in the nonlinear RMF model.

\section{RESULTS AND DISCUSSIONS}

Using these models, we have studied the isospin-dependent properties of asymmetric nuclear matter. In the following, we focus on results regarding the nuclear symmetry energy, nuclear symmetry potential, isospin splitting of nucleon effective mass, and isospin-dependent nucleon scalar density in asymmetric nuclear matter. For the different versions of the RMF model considered in the present work, we mainly consider parameter sets commonly and successfully used in nuclear structure studies. In particular, we select the parameter sets NL1 [103], NL2 [103], NL3 [104], NL-SH [105], TM1 [106], PK1 [107], FSU-Gold [77], HA [108], NL $\rho$ [101], NL $\rho \delta$ [101] for the nonlinear RMF model; TW99 [47], DD-ME1 [109], DD-ME2 [110], PKDD [107], DD [94], DD-F [111], and DDRH-corr [48] for the density-dependent RMF model; and PC-F1 [50], PC-F2 [50], PC-F3 [50], PC-F4 [50], PC-LA [50], and FKVW [54] for the point-coupling RMF model. There are a total of 23 parameter sets, most of which can describe reasonably well the binding energies and charge radii of a large number of nuclei in the periodic table except for parameter set HA, for which to our knowledge there are no calculations for finite nuclei.

We note that all selected parameter sets include the isovector-vector channel involving either the isovector-vector $\rho$ meson or the isovector-vector interaction vertices in the Lagrangian. The HA parameter set further includes the isovector-scalar meson field $\vec{\delta}$ and fits successfully some results obtained from the more microscopic DBHF approach [108]. The parameter sets NL $\rho \delta$ and DDRH-corr also include the isovector-scalar meson field $\vec{\delta}$, while PC-F2, PC-F4, PC-LA, and FKVW include the isovector-scalar interaction vertices. The parameter sets $\mathrm{NL} \rho \delta$ and $\mathrm{NL} \rho$ are obtained by fitting the empirical properties of asymmetric nuclear matter [101] and describe reasonably well the binding energies 
and charge radii of a large number of nuclei [112]. For the DDRH-corr, its parameters are determined from the density-dependent meson-nucleon vertices extracted from the self-energies of asymmetric nuclear matter calculated in the microscopic DBHF approach with momentum corrections, and it reproduces satisfactorily the properties of finite nuclei and the EOS from the DBHF approach [48]. In the parameter sets PC-F1, PC-F2, PC-F3, PC-F4, and PC-LA for the nonlinear point-coupling model, their coupling constants are determined in a self-consistent procedure that solves the model equations for representative nuclei simultaneously in a generalized nonlinear least-squares adjustment algorithm [50]. The parameters of set FKVW for the density-dependent pointcoupling model are determined by the constraints derived from the finite-density QCD sum rules, in-medium chiral perturbation theory, and experimental data of a number of finite nuclei [54].

\section{A. Nuclear symmetry energy}

Figure 1 displays the density dependence of the nuclear symmetry energy $E_{\text {sym }}(\rho)$ for the 23 parameter sets in the nonlinear, density-dependent, and point-coupling RMF models. For comparison, we also show in Fig. 1 results from the phenomenological parametrization of the momentumdependent nuclear mean-field potential based on the Gogny effective interaction [25], i.e., the MDI interactions with $x=-1$ (open squares) and 0 (solid squares), where different $x$ values correspond to different density dependence of the nuclear symmetry energy but keep other properties of the nuclear EOS the same [56] (see the Appendix for details).

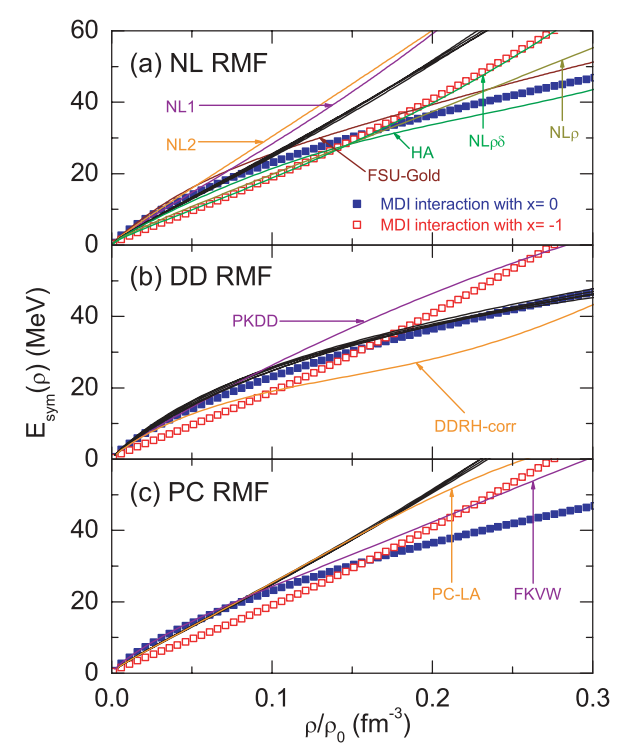

FIG. 1. (Color online) Density dependence of the nuclear symmetry energy $E_{\text {sym }}(\rho)$ for the parameter sets NL1, NL2, NL3, NL-SH, TM1, PK1, FSU-Gold, HA, NL $\rho$, and NL $\rho \delta$ in the nonlinear RMF model (a); TW99, DD-ME1, DD-ME2, PKDD, DD, DD-F, and DDRH-corr in the density-dependent RMF model (b); and PC-F1, PC-F2, PC-F3, PC-F4, PC-LA, and FKVW in the point-coupling RMF model (c). For comparison, results from the MDI interaction with $x=-1$ and 0 are also shown.
From analyzing the isospin diffusion data from NSCL/MSU using the IBUU04 transport model with in-medium $N N$ cross sections, it has been found that the MDI interactions with $x=-1$ and 0 give, respectively, the upper and lower bounds for the stiffness of the nuclear symmetry energy at densities up to about $1.2 \rho_{0}[56,57]$.

It is seen from Fig. 1 that the density dependence of symmetry energy varies drastically among different interactions. In the nonlinear RMF model, while the dependence on density is almost linear for most parameter sets, it is much softer for the parameter sets FSU-Gold and HA. The softening of the symmetry energy from the latter two parameter sets is due to the mixed isoscalar-isovector couplings $\Lambda_{S}$ and $\Lambda_{V}[67,100]$ which modifies the density dependence of symmetry energy as seen in Eq. (44). For parameter set NL $\rho \delta$, it gives a symmetry energy that depends linearly on density at low densities but becomes stiffer at high densities due to inclusion of the isovector-scalar $\delta$ meson. The approximate linear density-dependent behavior of the symmetry energy for other parameter sets in the nonlinear RMF model can also be understood from Eq. (44), which shows that the symmetry energy at high densities is dominated by the potential energy that is proportional to the baryon density if the mixed isoscalarisovector coupling and the isovector-scalar $\delta$ meson are not included in the model.

The density dependence of the symmetry energy in the density-dependent RMF model is essentially determined by the density dependence of the coupling constants $\Gamma_{\rho}$ and $\Gamma_{\delta}$ of isovector mesons. Most parameter sets in this case give similar symmetry energies except parameter sets PKDD and DDRH-corr. Compared with other parameter sets in the density-dependent RMF model, PKDD gives a very large while DDRH-corr gives a very small value for the symmetry energy at saturation density. For point-coupling models, all parameter sets (PC-F1, PC-F2, PC-F3, PC-F4, and PC-LA) in the nonlinear point-coupling RMF model predict almost linearly density-dependent symmetry energies, while the parameter set FKVW in the density-dependent point-coupling RMF model gives a somewhat softer symmetry energy.

Figure 1 thus shows that only a few parameter sets can give symmetry energies that are consistent with the constraint from the isospin diffusion data in heavy-ion collisions, which is given by results from the MDI interactions with $x=-1$ and 0 . The main reason for this is that most parameter sets in the RMF model have saturation densities and symmetry energies at their saturation densities which are significantly different from the empirical saturation density of $0.16 \mathrm{fm}^{-3}$ and symmetry energy of $31.6 \mathrm{MeV}$ at this saturation density. To show this more clearly, we list in Table I the bulk properties of nuclear matter at saturation density: the binding energy per nucleon $-B / A(\mathrm{MeV})$, the saturation density of symmetric nuclear matter $\rho_{0}\left(\mathrm{fm}^{-3}\right)$, the incompressibility of symmetric nuclear matter $K_{0}(\mathrm{MeV})$, the symmetry energy $E_{\text {sym }}\left(\rho_{0}\right)(\mathrm{MeV}), K_{\text {sym }}$ $(\mathrm{MeV}), L(\mathrm{MeV})$, and $K_{\text {asy }}(\mathrm{MeV})$ using the 23 parameter sets in the nonlinear, density-dependent, and point-coupling RMF models. It is seen that these parameter sets give saturation densities varying from $\rho_{0}=0.145$ to $\rho_{0}=0.180 \mathrm{fm}^{-3}$ and nuclear symmetry energies $E_{\text {sym }}\left(\rho_{0}\right)(\mathrm{MeV})$ ranging from 26.1 to $44.0 \mathrm{MeV}$. 
To remove the effect due to differences in the saturation densities among different parameter sets, we show in Fig. 2 both the symmetry energy $E_{\text {sym }}(\rho)$ and the symmetry energy scaled by its value at corresponding saturation density, i.e., $E_{\text {sym }}(\rho) / E_{\text {sym }}\left(\rho_{0}\right)$ as functions of the scaled baryon density $\rho / \rho_{0}$ for different parameter sets. For comparison, we also plot in the inset in panel (b) of Fig. 2 the symmetry energy $E_{\text {sym }}(\rho)$ as a function of the baryon density $\rho$ without scaling. It is seen that more parameter sets among the 23 sets become consistent with the constraint from the isospin diffusion data in heavy-ion collisions after scaling the baryon density by the saturation density; and with further scaling of the symmetry energy by its value at corresponding saturation density, most of the parameter sets agree with the constraint from the isospin diffusion data. It is also interesting to see from the inset in Fig. 2 that most of the parameter sets obtained from fitting the properties of finite nuclei give roughly the same value of about $26 \mathrm{MeV}$ for the nuclear symmetry energy at the same baryon density of $\rho=0.1 \mathrm{fm}^{-3}$. This interesting feature is very similar to that found with Skyrme interactions [65,71]. It implies that the constraint on the symmetry energy from fitting the properties of finite nuclei is particularly sensitive to the nuclear properties at lower densities, i.e., at density slightly above half-saturation density.

For the density dependence of the nuclear symmetry energy around saturation density, a more reasonable and physically meaningful comparison is through the values of $L$ and $K_{\text {asy }}$ given by these parameter sets, since the $L$ parameter is correlated linearly to the neutron-skin thickness of finite nuclei while the $K_{\text {asy }}$ parameter determines the isotopic dependence of the GMR for a fixed element. From Table I, we see that the values of $L, K_{\text {sym }}$, and $K_{\text {asy }}$ vary drastically, and they are in the range of $51 \sim 140 \mathrm{MeV},-140 \sim 143 \mathrm{MeV}$, and $-750 \sim-151$ $\mathrm{MeV}$, respectively. The extracted values of $L=88 \pm 25 \mathrm{MeV}$ and $K_{\text {asy }}=-500 \pm 50 \mathrm{MeV}$ from the isospin diffusion data, $L \approx 65 \mathrm{MeV}$ and $K_{\text {asy }} \approx-453 \mathrm{MeV}$ from the isoscaling data, and $K_{\text {asy }}=-550 \pm 100 \mathrm{MeV}$ from the isotopic dependence of the GMR in even- $A$ Sn isotopes give a rather stringent constraint on the density dependence of the nuclear symmetry energy and thus put strong constraints on the nuclear effective interactions as well. To see this constraint more clearly, we collect in Fig. 3 the values of $L$ and $K_{\text {asy }}$ obtained from the 23 parameter sets in the nonlinear, density-dependent, and point-coupling RMF models together with the constraints from the isospin diffusion data, isoscaling data, and the isotopic dependence of the GMR in even- $A$ Sn isotopes. From Fig. 3 as well as Table I, we see clearly that among the 23 parameter sets considered here, only six sets, i.e., TM1, NL $\rho$, NL $\rho \delta$, PKDD, PC-LA, and FKVW, have nuclear symmetry energies that are consistent with the extracted $L$ value of $88 \pm 25$ $\mathrm{MeV}$ while 15 sets, i.e., NL3, NL-SH, TM1, PK1, HA, NL $\rho$, NL $\rho \delta$, TW99, PKDD, DD-F, PC-F1, PC-F2, PC-F3, PC-F4, and FKVW, have nuclear symmetry energies that are consistent with the extracted $K_{\text {asy }}$ value of $-500 \pm 50$ or

TABLE I. Bulk properties of nuclear matter at the saturation point: $-B / A(\mathrm{MeV}), \rho_{0}\left(\mathrm{fm}^{-3}\right), K_{0}(\mathrm{MeV})$, $E_{\text {sym }}\left(\rho_{0}\right)(\mathrm{MeV}), K_{\text {sym }}(\mathrm{MeV}), L(\mathrm{MeV})$, and $K_{\text {asy }}(\mathrm{MeV})$ using the 23 parameter sets in the nonlinear, density-dependent, and point-coupling RMF models. The last column gives the references for corresponding parameter sets.

\begin{tabular}{lccccrrrr}
\hline \hline Model & $-B / A$ & $\rho_{0}$ & $K_{0}$ & $E_{\text {sym }}$ & $L$ & $K_{\text {sym }}$ & $K_{\text {asy }}$ & Ref. \\
\hline NL1 & 16.4 & 0.152 & 212 & 43.5 & 140 & 143 & -697 & {$[103]$} \\
NL2 & 17.0 & 0.146 & 401 & 44.0 & 130 & 20 & -750 & {$[103]$} \\
NL3 & 16.2 & 0.148 & 271 & 37.3 & 118 & 100 & -608 & {$[104]$} \\
NL-SH & 16.3 & 0.146 & 356 & 36.1 & 114 & 80 & -604 & {$[105]$} \\
TM1 & 16.3 & 0.145 & 281 & 36.8 & 111 & 34 & -632 & {$[106]$} \\
PK1 & 16.3 & 0.148 & 282 & 37.6 & 116 & 55 & -641 & {$[107]$} \\
FSUGold & 16.3 & 0.148 & 229 & 32.5 & 60 & -52 & -412 & {$[77]$} \\
HA & 15.6 & 0.170 & 233 & 30.7 & 55 & -135 & -465 & {$[108]$} \\
NL $\rho$ & 16.1 & 0.160 & 240 & 30.3 & 85 & 3 & -507 & {$[101]$} \\
NL $\rho \delta$ & 16.1 & 0.160 & 240 & 30.7 & 103 & 127 & -491 & {$[101]$} \\
TW99 & 16.2 & 0.153 & 241 & 32.8 & 55 & -124 & -454 & {$[47]$} \\
DD-ME1 & 16.2 & 0.152 & 245 & 33.1 & 55 & -101 & -431 & {$[109]$} \\
DD-ME2 & 16.1 & 0.152 & 251 & 32.3 & 51 & -87 & -393 & {$[110]$} \\
PKDD & 16.3 & 0.150 & 263 & 36.9 & 90 & -80 & -620 & {$[107]$} \\
DD & 16.0 & 0.149 & 241 & 31.7 & 56 & -95 & -431 & {$[94]$} \\
DD-F & 16.0 & 0.147 & 223 & 31.6 & 56 & -140 & -476 & {$[111]$} \\
DDRH-corr & 15.6 & 0.180 & 281 & 26.1 & 51 & 155 & -151 & {$[48]$} \\
PC-F1 & 16.2 & 0.151 & 255 & 37.8 & 117 & 75 & -627 & {$[50]$} \\
PC-F2 & 16.2 & 0.151 & 256 & 37.6 & 116 & 65 & -631 & {$[50]$} \\
PC-F3 & 16.2 & 0.151 & 256 & 38.3 & 119 & 74 & -640 & {$[50]$} \\
PC-F4 & 16.2 & 0.151 & 255 & 37.7 & 119 & 98 & -616 & {$[50]$} \\
PC-LA & 16.1 & 0.148 & 263 & 37.2 & 108 & -61 & -709 & {$[50]$} \\
FKVW & 16.2 & 0.149 & 379 & 33.1 & 80 & 11 & -469 & {$[54]$} \\
\hline \hline
\end{tabular}




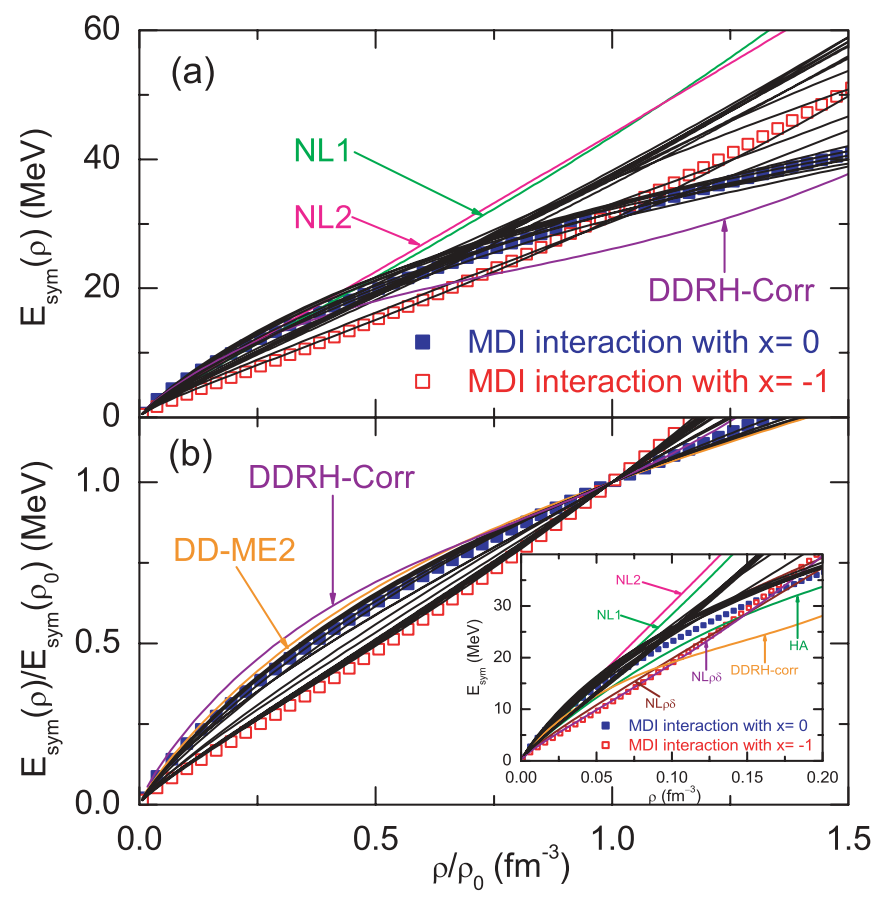

FIG. 2. (Color online) Symmetry energy $E_{\text {sym }}(\rho)$ (a) and the scaled symmetry energy $E_{\text {sym }}(\rho) / E_{\text {sym }}\left(\rho_{0}\right)$ (b) as functions of the scaled baryon density $\rho / \rho_{0}$ for the 23 parameter sets in the nonlinear, density-dependent, and point-coupling RMF models. Results of the MDI interaction with $x=-1$ and 0 are also included for comparison. The inset in panel (b) shows the symmetry energy $E_{\text {sym }}(\rho)$ as a function of the baryon density $\rho$ without scaling.

$-550 \pm 100 \mathrm{MeV}$. Among the latter 15 sets, only six sets, i.e., HA, NL $\rho$, NL $\rho \delta$, TW99, DD-F, and FKVW, are consistent with $K_{\text {asy }}=-500 \pm 50 \mathrm{MeV}$. It is interesting to see that most parameter sets in the nonlinear and point-coupling RMF

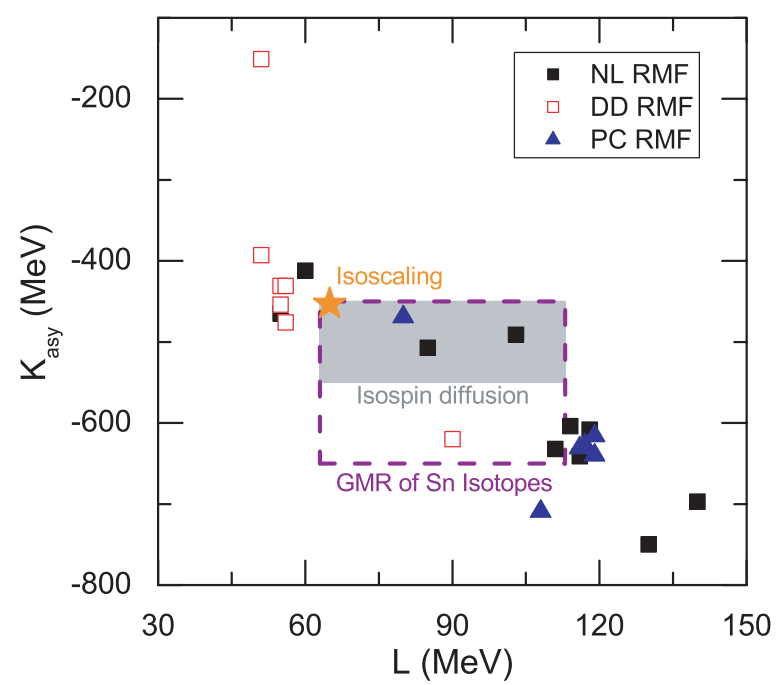

FIG. 4. (Color online) Correlation between $L$ and $K_{\text {asy }}$ for the 23 parameter sets in the nonlinear, density-dependent, and pointcoupling RMF models. The constraints from the isospin diffusion data (shaded band), the isoscaling data (stars), and the isotopic dependence of the GMR in even- $A$ Sn isotopes (dashed rectangle with $L$ constrained by the isospin diffusion data) are also included.

models predict stiffer symmetry energies (i.e., larger values for the $L$ parameter and larger magnitudes for $K_{\text {asy }}$ ), while those in the density-dependent RMF model give softer symmetry energies (i.e., smaller values for the $L$ parameter and smaller magnitudes for $K_{\text {asy }}$ ).

We also see from Table I that only five parameter sets, i.e., TM1, NL $\rho, \mathrm{NL} \rho \delta$, PKDD, and FKVW, have nuclear symmetry energies that are consistent with the extracted values for both $L$ and $K_{\text {asy }}(-500 \pm 50$ or $-550 \pm 100 \mathrm{MeV})$. This can be seen more clearly in Fig. 4 where the correlation

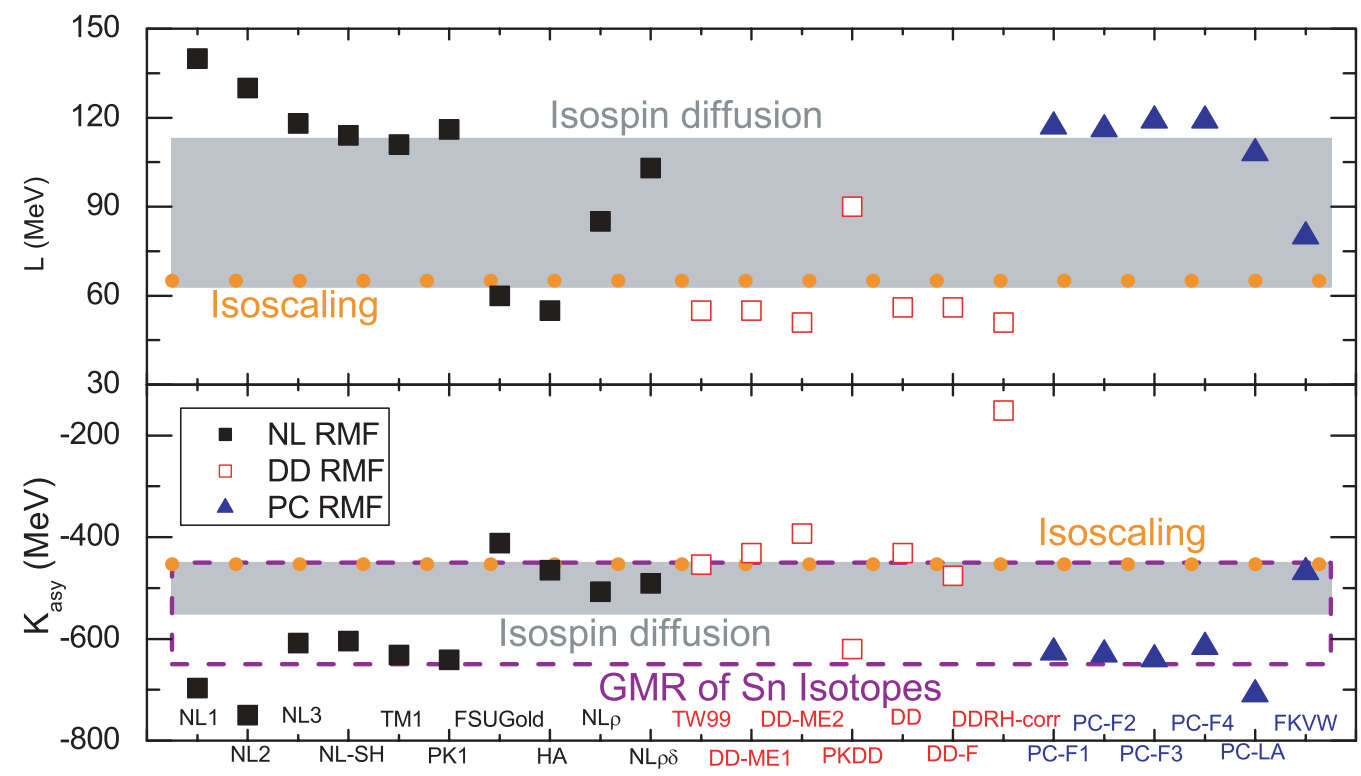

FIG. 3. (Color online) Values of $L$ and $K_{\text {asy }}$ for the 23 parameter sets in the nonlinear, density-dependent, and point-coupling RMF models. The constraints from the isospin diffusion data (shaded band), the isoscaling data (solid circles), and the isotopic dependence of the GMR in even- $A$ Sn isotopes (dashed rectangle) are also included. 
between $L$ and $K_{\text {asy }}$ is displayed for the 23 parameter sets together with the constraints from the isospin diffusion data, the isoscaling data, and the isotopic dependence of the GMR in even- $A \mathrm{Sn}$ isotopes. Figure 4 further shows that there exists an approximately linear correlation between $L$ and $K_{\text {asy }}$, i.e., a larger $L$ leads to a larger magnitude for $K_{\text {asy }}$. A similar approximately linear correlation between $L$ and $K_{\text {asy }}$ has also been observed in Ref. [56] for the phenomenological MDI interactions, and this correlation can be understood from Eq. (7) which shows that the value of $K_{\text {asy }}$ is more sensitive to the value of $L$ than to that of $K_{\text {sym }}$.

The above comparisons thus indicate that the extracted values of $L=88 \pm 25 \mathrm{MeV}$ and $K_{\text {asy }}=-500 \pm 50 \mathrm{MeV}$ from the isospin diffusion data, $L \approx 65 \mathrm{MeV}$ and $K_{\text {asy }} \approx$ $-453 \mathrm{MeV}$ from the isoscaling data, and $K_{\text {asy }}=-550 \pm$ $100 \mathrm{MeV}$ from the isotopic dependence of the GMR in even- $A$ $\mathrm{Sn}$ isotopes indeed put a very stringent constraint on the values of the parameters in different RMF models. The fact that most of the 23 parameter sets considered in the present work give symmetry energies that are inconsistent with the constraints of $L=88 \pm 25 \mathrm{MeV}$ and $K_{\text {asy }}=-500 \pm 50$ or $-550 \pm$ $100 \mathrm{MeV}$ is probably related to the rather limited flexibility in the parametrization of the isovector channel in all RMF models. They are also probably connected to the fact that most of the parameter sets are obtained from fitting properties of finite nuclei, which are mostly near the $\beta$-stability line and thus are not well constrained by the isospin-dependent properties of nuclear EOS. Also, we are interested here in the density-dependent behavior of the symmetry energy around saturation density, as both $L$ and $K_{\text {asy }}$ are defined at saturation density, while the behavior of the nuclear EOS at subsaturation density may be more relevant when the parameter sets are obtained from fitting the properties of finite nuclei.

\section{B. Nuclear symmetry potential}

Using the parameter sets NL1, NL2, NL3, NL-SH, TM1, PK1, FSU-Gold, HA, NL $\rho$, and NL $\rho \delta$ in the nonlinear RMF model, we have evaluated the energy dependence of the three different nucleon optical potentials, i.e., the Schrödingerequivalent potential $U_{\text {SEP }}[\mathrm{Eq}$. (10)], the optical potential from the difference between the total energy of a nucleon in nuclear medium and its energy at the same momentum in free space $U_{\text {OPT }}$ [Eq. (12)], and the optical potential based on the second-order Dirac equation $U_{\text {SOD }}$ [Eq. (15)], at a fixed baryon density $\rho_{B}=0.16 \mathrm{fm}^{-3}$ (roughly corresponding to the saturation densities obtained from various RMF models). For their corresponding symmetry potentials $U_{\mathrm{sym}}^{\mathrm{SEP}}, U_{\mathrm{sym}}^{\mathrm{OPT}}$, and $U_{\text {sym }}^{\text {SOD }}$, we have evaluated instead their dependence on the nucleon momentum in asymmetric nuclear matter at baryon density $\rho_{B}=0.16 \mathrm{fm}^{-3}$ and with isospin asymmetry $\alpha=0.5$. We note that in contrast to the energy dependence of the nuclear symmetry potential, the momentum dependence of the nuclear symmetry potential is almost independent of the isospin asymmetry of nuclear matter. These results are shown in Fig. 5. Corresponding results for the parameter sets TW99, DD-ME1, DD-ME2, PKDD, DD, DD-F, and DDRH-corr in the density-dependent RMF model and for PC-F1, PC-F2,
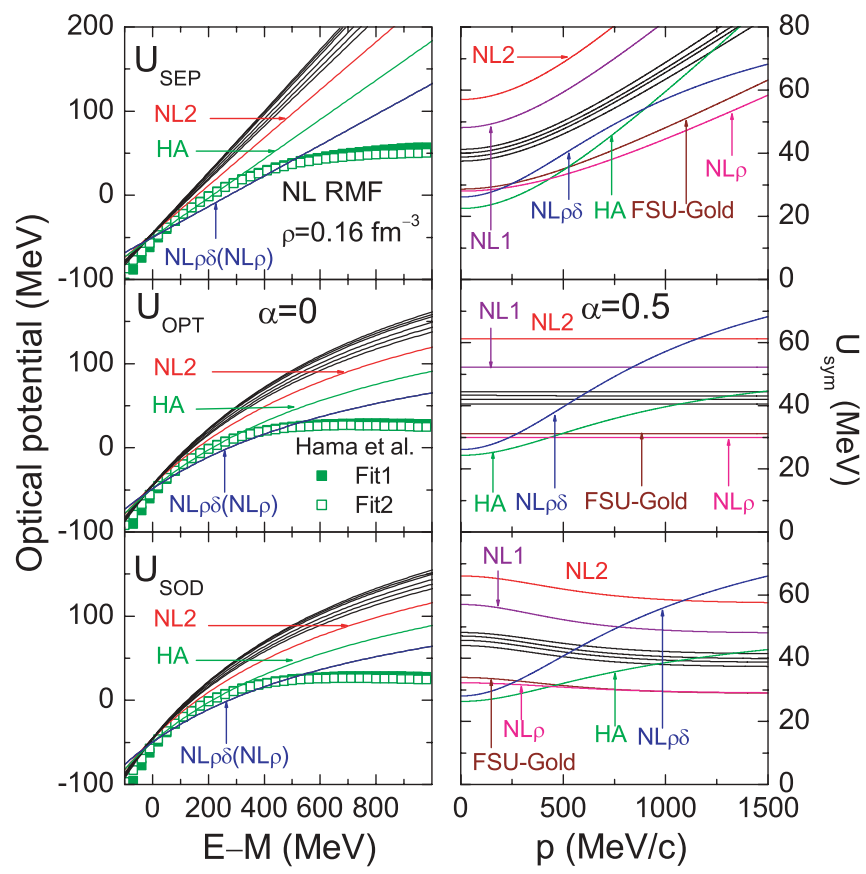

FIG. 5. (Color online) Energy dependence of the three different nucleon optical potentials, i.e., $U_{\mathrm{SEP}}$ [Eq. (10)], $U_{\mathrm{OPT}}$ [Eq. (12)], and $U_{\text {SOD }}$ [Eq. (15)] (left panels) as well as their corresponding symmetry potentials $U_{\mathrm{sym}}^{\mathrm{SEP}}, U_{\mathrm{sym}}^{\mathrm{OPT}}$, and $U_{\mathrm{sym}}^{\mathrm{SOD}}$ as functions of momentum (right panels), at a fixed baryon density $\rho_{B}=0.16 \mathrm{fm}^{-3}$ for parameter sets NL1, NL2, NL3, NL-SH, TM1, PK1, FSU-Gold, HA, NL $\rho$, and NL $\rho \delta$ in the nonlinear RMF model. For comparison, the energy dependence of the real part of the optical potential in symmetric nuclear matter at saturation density extracted from two different fits of the proton-nucleus scattering data in the Dirac phenomenology are also included (left panels).

PC-F3, PC-F4, PC-LA, and FKVW in the point-coupling RMF model are shown in Figs. 6 and 7, respectively. For comparison, we also include in these figures results for the energy dependence of the real part of the different optical potentials in symmetric nuclear matter at saturation density that are extracted from the proton-nucleus scattering data based on the Dirac phenomenology [83,113].

We see that different optical potentials in symmetric nuclear matter at $\rho_{B}=0.16 \mathrm{fm}^{-3}$ exhibit similar energy dependence at low energies but have different behaviors at high energies. In particular, at high energies, $U_{\mathrm{SEP}}$ continues to increase linearly with energy while $U_{\mathrm{OPT}}$ and $U_{\mathrm{SOD}}$ seem to saturate at high energies and thus display a more satisfactory high-energy limit, similar to what is observed in the nuclear optical potential that is extracted from the experimental data based on the Dirac phenomenology. The critical energy at which the optical potential changes from negative to positive values is between about 130 and $270 \mathrm{MeV}$, depending on the parameter sets used. These features are easy to understand from the fact that the scalar and vector potentials are momentum/energy independent in the RMF models considered here. Analysis of experimental data from the proton-nucleus scattering in the Dirac phenomenology also indicates that the extracted different nucleon optical potentials in symmetric nuclear matter at normal nuclear density change from negative to 

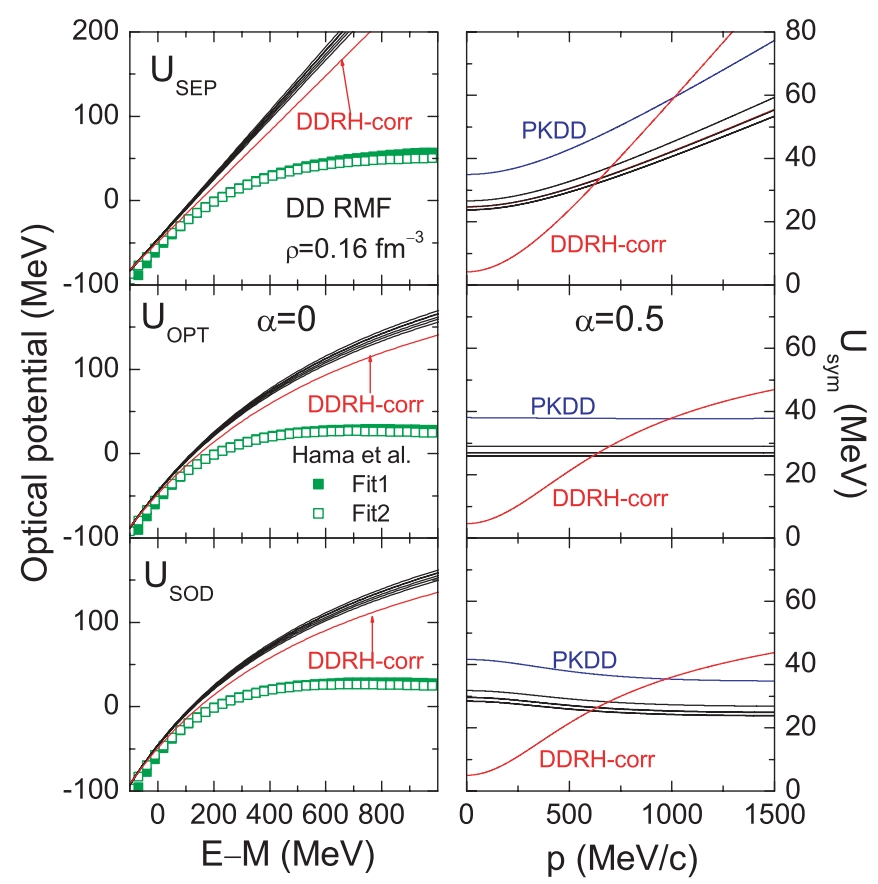

FIG. 6. (Color online) Same as Fig. 5, but for TW99, DD-ME1, DD-ME2, PKDD, DD, DD-F, and DDRH-corr in the densitydependent RMF models.

positive values at a nucleon energy of about $208 \mathrm{MeV}$. Furthermore, it is seen that the different optical potentials from all 23 parameter sets are consistent with the experimental data at lower energies, i.e., $E_{\text {kin }}<100-200 \mathrm{MeV}$, but are generally too repulsive at higher energies, especially for the Schrödinger-equivalent potential $U_{\text {SEP. }}$. These features imply that the RMF models with parameters fitted to the properties
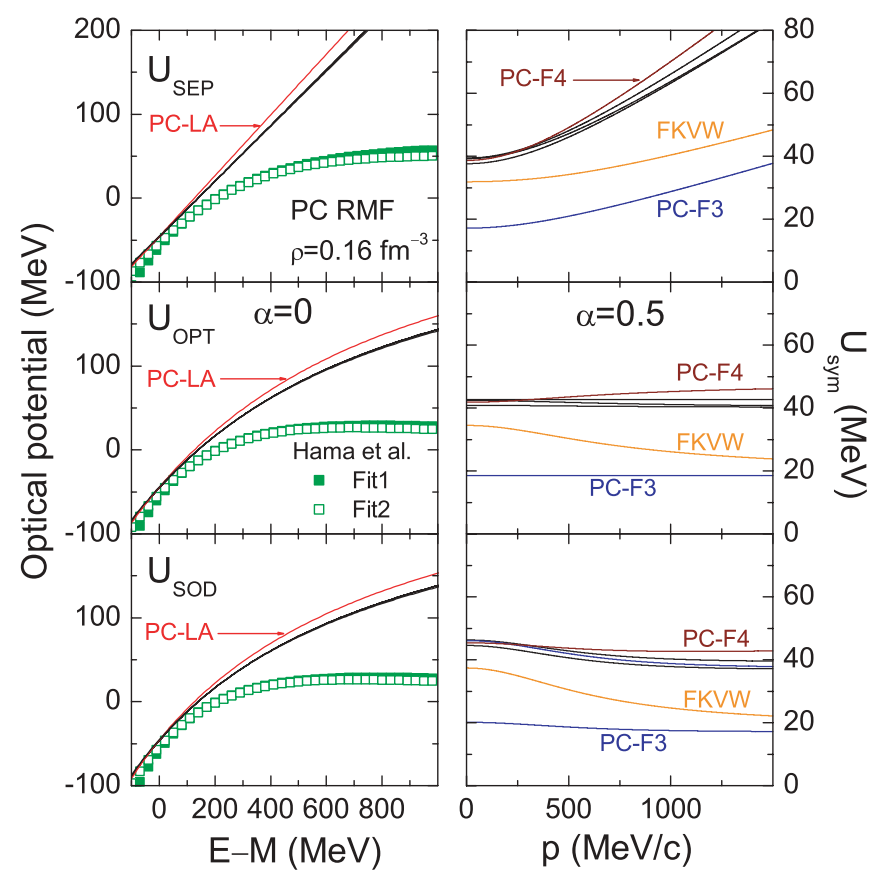

FIG. 7. (Color online) Same as Fig. 5, but for PC-F1, PC-F2, PCF3, PC-F4, PC-LA, and FKVW in the point-coupling RMF models. of finite nuclei can only give reasonable descriptions of the low energy behavior of the isoscalar optical potentials. On the other hand, it should be mentioned that for optical potentials at high energies, contributions from dispersive processes such as dynamical polarization by inelastic excitations, inelastic isobar resonance excitation above the pion threshold, and particle production become important $[114,115]$. Including such continuum excitations is expected to improve significantly the high energy behavior of the optical potential [114]. Such studies are, however, beyond the RMF model based on the Hartree level as considered here.

For the momentum dependence of the symmetry potential, all 23 parameter sets display similar behaviors in $U_{\text {sym }}^{\mathrm{SEP}}$, i.e., increasing with momentum, albeit at different rates. This can be qualitatively understood as follows. Expressing Eq. (10) as

$$
U_{\mathrm{SEP}, \tau}=\frac{1}{2 M_{\tau}}\left[E_{\tau}^{2}-\left(M_{\tau}^{2}+\vec{p}^{2}\right)\right],
$$

and neglecting the difference in neutron and proton masses, we can rewrite Eq. (11) as

$$
\begin{aligned}
U_{\text {sym }}^{\mathrm{SEP}}= & \frac{E_{n}^{2}-E_{p}^{2}}{4 M_{\tau} \alpha} \\
= & \frac{1}{4 M_{\tau} \alpha}\left[\left(\Sigma_{n}^{0}\right)^{2}+2 \Sigma_{n}^{0} \sqrt{\vec{p}^{2}+\left(M_{n}+\Sigma_{n}^{S}\right)^{2}}\right. \\
& +\left(M_{n}+\Sigma_{n}^{S}\right)^{2}-\left(\Sigma_{p}^{0}\right)^{2}-2 \Sigma_{p}^{0} \sqrt{\vec{p}^{2}+\left(M_{p}+\Sigma_{p}^{S}\right)^{2}} \\
& \left.-\left(M_{p}+\Sigma_{p}^{S}\right)^{2}\right]=\frac{1}{4 M_{\tau} \alpha}\left[\left(\Sigma_{n}^{0}\right)^{2}-\left(\Sigma_{p}^{0}\right)^{2}\right. \\
& +\left(M_{\text {Dirac }, n}^{*}\right)^{2}-\left(M_{\text {Dirac }, p}^{*}\right)^{2}+2 \Sigma_{n}^{0} \sqrt{\vec{p}^{2}+\left(M_{\text {Dirac }, n}^{*}\right)^{2}} \\
& \left.-2 \Sigma_{p}^{0} \sqrt{\vec{p}^{2}+\left(M_{\text {Dirac }, p}^{*}\right)^{2}}\right] .
\end{aligned}
$$

In the simple case of the nonlinear RMF model without the isovector-scalar $\delta$ meson, the neutron Dirac mass is the same as that of the proton. In this case, $U_{\text {sym }}^{\mathrm{SEP}}$ is reduced to

$$
\begin{aligned}
U_{\text {sym }}^{\mathrm{SEP}}= & \frac{1}{4 M_{\tau} \alpha}\left[\left(\Sigma_{n}^{0}\right)^{2}-\left(\Sigma_{p}^{0}\right)^{2}+2\left(\Sigma_{n}^{0}-\Sigma_{p}^{0}\right)\right. \\
& \left.\times \sqrt{\vec{p}^{2}+\left(M_{\text {Dirac }}^{*}\right)^{2}}\right] .
\end{aligned}
$$

Since it can be shown from Eqs. (31), (33), and (39) that

$$
\Sigma_{n}^{0}-\Sigma_{p}^{0}=2\left(\frac{g_{\rho}}{m_{\rho}}\right)^{2}\left(\rho_{n}-\rho_{p}\right),
$$

we thus have $\Sigma_{n}^{0}>\Sigma_{p}^{0}$ and an increase of $U_{\text {sym }}^{\text {SEP }}$ with the momentum of a nucleon in neutron-rich nuclear matter. The same argument applies to density-dependent RMF models and to point-coupling models if the coupling constant $\alpha_{T V}$ or $G_{T V}$ in the point-coupling models is positive (at saturation density) so that the potential energy part of the symmetry energy at saturation density is also positive.

For $U_{\text {sym }}^{\mathrm{OPT}}$, whether it increases or deceases with nucleon momentum depends on the isospin splitting of the nucleon scalar self-energy (scalar potential) or Dirac mass in neutronrich nuclear matter. This can be seen from Eq. (14) if it is 
reexpressed as

$$
\begin{aligned}
U_{\mathrm{sym}}^{\mathrm{OPT}}= & \frac{E_{n}-E_{p}}{2 \alpha} \\
= & \frac{1}{2 \alpha}\left[\Sigma_{n}^{0}-\Sigma_{p}^{0}+\sqrt{\vec{p}^{2}+\left(M_{n}+\Sigma_{n}^{S}\right)^{2}}\right. \\
& \left.-\sqrt{\vec{p}^{2}+\left(M_{p}+\Sigma_{p}^{S}\right)^{2}}\right] \\
= & \frac{1}{2 \alpha}\left[\Sigma_{n}^{0}-\Sigma_{p}^{0}+\sqrt{\vec{p}^{2}+\left(M_{\text {Dirac }, n}^{*}\right)^{2}}\right. \\
& \left.-\sqrt{\vec{p}^{2}+\left(M_{\text {Dirac }, p}^{*}\right)^{2}}\right] .
\end{aligned}
$$

We note that $U_{\text {sym }}^{\mathrm{OPT}}$ increases with momentum for the parameter sets HA, NL $\rho \delta$, DDRH-corr, and PC-F4, while the opposite behavior is observed for the parameter sets PC-F2, PC-LA, and FKVW.

The momentum dependence of $U_{\mathrm{sym}}^{\mathrm{SOD}}$ is similar to that of $U_{\text {sym }}^{\mathrm{OPT}}$ if we rewrite Eq. (16) as

$$
\begin{aligned}
U_{\mathrm{sym}}^{\mathrm{SOD}} & =\frac{E_{n}-E_{p}-\left(M_{\tau}^{2}+\vec{p}^{2}\right)\left(\frac{1}{E_{n}}-\frac{1}{E_{n}}\right)}{4 \alpha} \\
& =\frac{U_{\mathrm{sym}}^{\mathrm{OPT}}}{2}-\frac{\left(M_{\tau}^{2}+\vec{p}^{2}\right)\left(\frac{1}{E_{n}}-\frac{1}{E_{n}}\right)}{4 \alpha} .
\end{aligned}
$$

In this case, $U_{\mathrm{sym}}^{\mathrm{SOD}}$ increases with nucleon momentum for the parameter sets HA, NL $\rho \delta$, and DDRH-corr, while it decreases for other parameter sets considered here.

In Ref. [79], it has been argued that it is the Schrödingerequivalent potential $U_{\text {SEP }}$ [Eq. (10)] and thus its corresponding symmetry potential $U_{\text {sym }}^{\text {SEP }}$ that should be compared with the results from nonrelativistic models. As discussed before, the experimental data indicate that the nuclear symmetry potential at nuclear matter saturation density, i.e., the Lane potential $U_{\text {Lane}}$, clearly decreases at low energies (beam energy $E_{\text {kin }}$ up to about $100 \mathrm{MeV}$ and corresponding momentum values ranging from about 300 to $470 \mathrm{MeV} / \mathrm{c}$ ), which is obviously contradictory to the results for $U_{\text {sym }}^{\text {SEP }}$ from all 23 parameter sets considered here. On the other hand, $U_{\mathrm{sym}}^{\mathrm{OPT}}$ and $U_{\mathrm{sym}}^{\mathrm{SOD}}$ for some parameter sets can decrease with nucleon momentum, which is qualitatively consistent with experimental results.

For nucleons with momenta less than about $250-300 \mathrm{MeV} / \mathrm{c}$ or $E_{\text {kin }}<0$, although the observed increase of $U_{\text {sym }}^{\text {SEP }}$ with momentum for all 23 parameter sets, and $U_{\text {sym }}^{\mathrm{OPT}}$ as well as $U_{\text {sym }}^{\text {SOD }}$ with some parameter sets, seems to be consistent with the results from the microscopic DBHF [17], the extended BHF with three-body forces [24], and chiral perturbation theory calculations [89], i.e., the symmetry potential stays as a constant or slightly increases with momentum before decreasing at high momenta, it fails to describe the high momentum/energy behaviors of the nuclear symmetry potential extracted from nucleon-nucleus scattering experiments and $(p, n)$ charge exchange reactions at beam energies up to about $100 \mathrm{MeV}$.

We note that in studies based on the relativistic impulse approximation with empirical $N N$ scattering amplitude and the nuclear scalar and vector densities from the RMF model, the Schrödinger-equivalent nuclear symmetry potential at fixed
TABLE II. Values of different nucleon effective masses in symmetric nuclear matter at saturation density using the 23 parameter sets in the nonlinear, density-dependent, and point-coupling RMF models. The last column gives the references for corresponding parameter sets.

\begin{tabular}{lcccccc}
\hline \hline Model & $\frac{M_{\text {Dirac }}^{*}}{M}$ & $\frac{M_{\text {Landau }}^{*}}{M}$ & $\frac{M_{\text {Lorentz }}^{*}}{M}$ & $\frac{M_{\text {OPT }}^{*}}{M}$ & $\frac{M_{\text {SOD }}^{*}}{M}$ & Ref. \\
\hline NL1 & 0.57 & 0.64 & 0.65 & 0.61 & 0.59 & {$[103]$} \\
NL2 & 0.67 & 0.72 & 0.74 & 0.70 & 0.68 & {$[103]$} \\
NL3 & 0.60 & 0.66 & 0.67 & 0.63 & 0.61 & {$[104]$} \\
NL-SH & 0.60 & 0.66 & 0.67 & 0.63 & 0.61 & {$[105]$} \\
TM1 & 0.63 & 0.69 & 0.71 & 0.67 & 0.65 & {$[106]$} \\
PK1 & 0.61 & 0.66 & 0.68 & 0.64 & 0.62 & {$[107]$} \\
FSUGold & 0.61 & 0.67 & 0.69 & 0.65 & 0.62 & {$[77]$} \\
HA & 0.68 & 0.74 & 0.75 & 0.71 & 0.69 & {$[108]$} \\
NL $\rho$ & 0.75 & 0.80 & 0.82 & 0.77 & 0.76 & {$[101]$} \\
NL $\rho \delta$ & 0.75 & 0.80 & 0.82 & 0.77 & 0.76 & {$[101]$} \\
TW99 & 0.55 & 0.62 & 0.64 & 0.60 & 0.57 & {$[47]$} \\
DD-ME1 & 0.58 & 0.64 & 0.66 & 0.62 & 0.59 & {$[109]$} \\
DD-ME2 & 0.57 & 0.63 & 0.65 & 0.61 & 0.59 & {$[110]$} \\
PKDD & 0.57 & 0.63 & 0.65 & 0.61 & 0.59 & {$[107]$} \\
DD & 0.56 & 0.63 & 0.64 & 0.61 & 0.58 & {$[94]$} \\
DD-F & 0.56 & 0.62 & 0.64 & 0.60 & 0.57 & {$[111]$} \\
DDRH- & 0.55 & 0.63 & 0.64 & 0.60 & 0.58 & {$[48]$} \\
corr & & & & & & \\
PC-F1 & 0.61 & 0.67 & 0.69 & 0.64 & 0.62 & {$[50]$} \\
PC-F2 & 0.61 & 0.67 & 0.69 & 0.64 & 0.62 & {$[50]$} \\
PC-F3 & 0.61 & 0.67 & 0.69 & 0.64 & 0.62 & {$[50]$} \\
PC-F4 & 0.61 & 0.67 & 0.69 & 0.64 & 0.62 & {$[50]$} \\
PC-LA & 0.58 & 0.64 & 0.65 & 0.61 & 0.59 & {$[50]$} \\
FKVW & 0.62 & 0.68 & 0.70 & 0.65 & 0.63 & {$[54]$} \\
\hline \hline
\end{tabular}

baryon density is found to decrease with increasing nucleon energy in the range of $100 \leqslant E_{\text {kin }} \leqslant 400 \mathrm{MeV}$ [93] and becomes essentially constant once the nucleon kinetic energy is greater than about $500 \mathrm{MeV}$ [90].

\section{Nucleon effective mass}

For the different nucleon effective masses in symmetric nuclear matter at saturation density, we show in Table II the results from the 23 parameter sets in the nonlinear, densitydependent, and point-coupling RMF models. It is seen that the values of $M_{\text {Dirac }}^{*} / M, M_{\text {Landau }}^{*} / M, M_{\text {Lorentz }}^{*} / M, M_{\mathrm{OPT}}^{*} / M$, and $M_{\mathrm{SOD}}^{*} / M$ are in the range of $0.55 \sim 0.75,0.62 \sim 0.80$, $0.64 \sim 0.80,0.60 \sim 0.77$, and $0.57 \sim 0.76$, respectively. The parameter sets NL2, HA, NL $\rho$, and $\mathrm{NL} \rho \delta$ seem to give too large values, i.e., $0.67,0.68,0.75$, and 0.75 , respectively, for the $M_{\text {Dirac }}^{*} / M$, as values in the range of $0.55 \sim 0.60$ are needed to describe reasonably the spin-orbit splitting in finite nuclei using the RMF models. On the other hand, the larger Dirac masses lead to larger Landau masses $M_{\text {Landau }}^{*} / M$ of 0.72 , $0.74,0.80$, and 0.80 , respectively, for the parameter sets NL2, $\mathrm{HA}, \mathrm{NL} \rho$, and $\mathrm{NL} \rho \delta$, which are consistent with the empirical constraint of $M_{\text {Landau }}^{*} / M=0.8 \pm 0.1$ [95-98].

The density dependence of the different nucleon effective masses in symmetric nuclear matter and corresponding isospin 

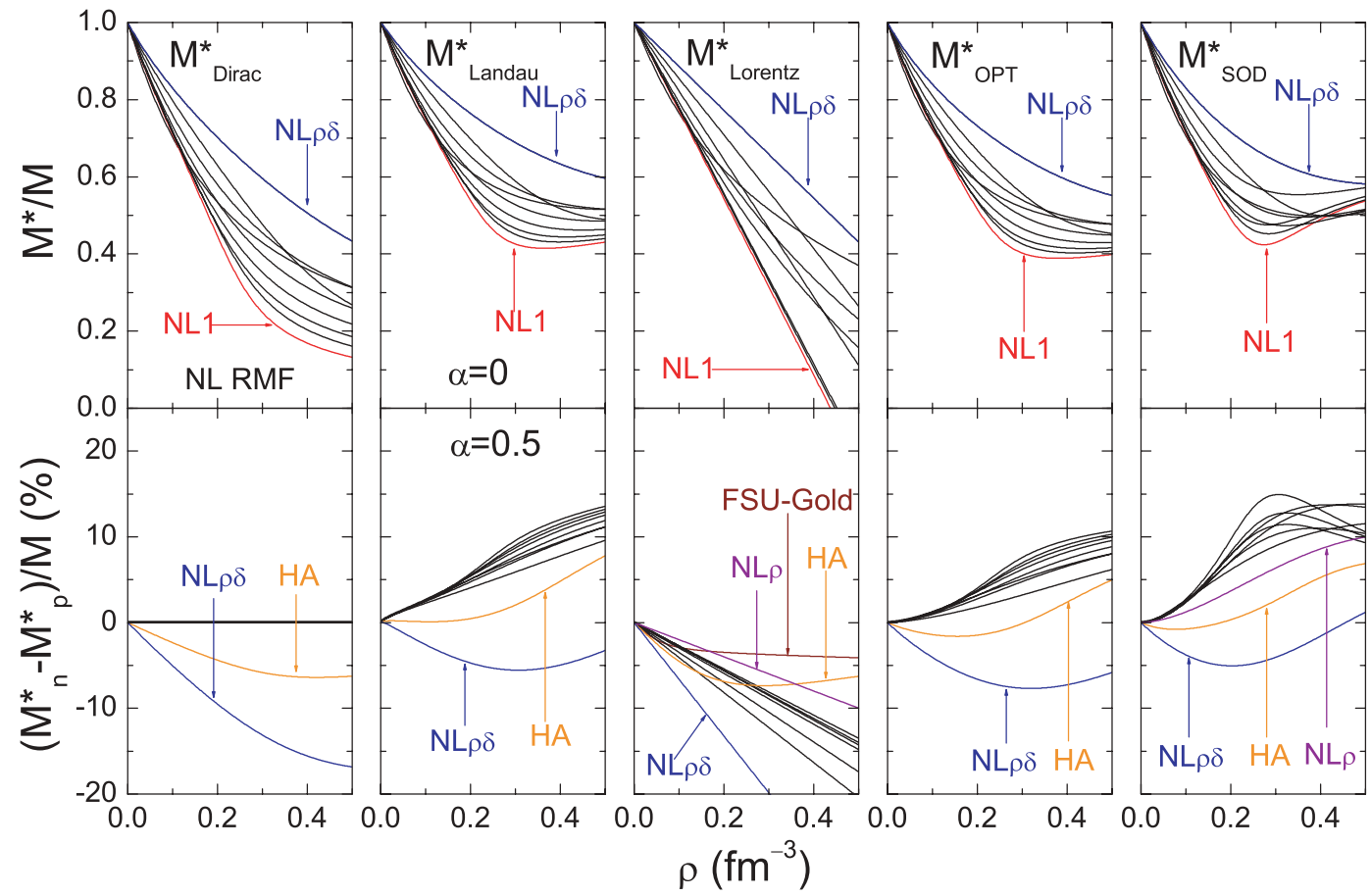

FIG. 8. (Color online) Density dependence of different nucleon effective masses, i.e., $M_{\mathrm{Dirac}}^{*} / M, M_{\mathrm{Landau}}^{*} / M, M_{\mathrm{Lorentz}}^{*} / M, M_{\mathrm{OPT}}^{*} / M$, and $M_{\mathrm{SOD}}^{*} / M$ in symmetric nuclear matter as well as their corresponding isospin splittings in neutron-rich nuclear matter with isospin asymmetry $\alpha=0.5$ for the parameter sets NL1, NL2, NL3, NL-SH, TM1, PK1, FSU-Gold, HA, NL $\rho$, and NL $\rho \delta$ in the nonlinear RMF model.

splitting $\left(M_{n}^{*}-M_{p}^{*}\right) / M$ in asymmetric nuclear matter with isospin asymmetry $\alpha=0.5$ are shown in Fig. 8 for the parameter sets NL1, NL2, NL3, NL-SH, TM1, PK1, FSUGold, HA, NL $\rho$, and NL $\rho \delta$ in the nonlinear RMF model. Figures 9 and 10 display the same results as in Fig. 8 but for the parameter sets TW99, DD-ME1, DD-ME2, PKDD, DD, DD-F, and DDRH-corr in the density-dependent RMF models and for PC-F1, PC-F2, PC-F3, PC-F4, PC-LA, and FKVW in the point-coupling RMF model, respectively. It is seen that different parameter sets in the nonlinear RMF model give significantly different density dependence for the nucleon effective masses while the different parameter sets in the density-dependent and point-coupling RMF models predict roughly the same density dependence for the nucleon effective masses, except that parameter set PC-LA gives very large values for the nucleon effective masses at high densities. This unusual behavior for PC-LA was also observed in Ref. [50], and it occurs because the coupling constant $\gamma_{S}$ for the higher-order interaction term in PC-LA is positive [49] and dominates at high density, leading thus to the very large nucleon effective mass.

For the Landau mass at a fixed baryon density, its value $M_{\text {Landau }}^{*} / M$ is generally larger than $M_{\text {Dirac }}^{*} / M$. This can be seen from Eq. (18) if it is rewritten as

$$
\begin{aligned}
M_{\text {Landau }, \tau}^{*} & =\left(E_{\tau}-\Sigma_{\tau}^{0}\right)=\sqrt{p_{F, \tau}^{2}+\left(M_{\tau}+\Sigma_{\tau}^{S}\right)^{2}} \\
& =\sqrt{p_{F, \tau}^{2}+M_{\text {Dirac }, \tau}^{* 2}},
\end{aligned}
$$

which shows that $M_{\text {Landau }, \tau}^{*} \geqslant M_{\text {Dirac }, \tau}^{*}$ if nucleon self-energies are independent of momentum/energy.
For the Lorentz mass, $M_{\mathrm{Lorentz}}^{*} / M$ depends almost linearly on density and thus has a stronger density dependence than the Dirac and Landau masses. We note from Eqs. (13) and (115) that Eq. (19) can be reduced to

$$
M_{\text {Lorentz, } \tau}^{*}=M_{\tau}-\Sigma_{\tau}^{0},
$$

if nucleon self-energies are independent of momentum/energy. Therefore, the density dependence of $M_{\text {Lorentz }}^{*}$ is determined uniquely by the density dependence of the nucleon vector selfenergy. In the nonlinear RMF model, most of the parameter sets (except for TM1, PK1, and FSU-Gold, which include the selfcoupling of the $\omega$ meson field) give a linear density dependence for $\Sigma_{\tau}^{0}$, leading thus to the observed linear density dependence of $M_{\mathrm{Lorentz}}^{*}$. As for the nonlinear density dependence of $M_{\text {Lorentz }}^{*}$ in the density-dependent RMF model and point-coupling models, it is due to the nonlinear density dependence of the coupling constant or the inclusion of higher-order couplings.

$M_{\mathrm{OPT}}^{*} / M$ and $M_{\mathrm{SOD}}^{*} / M$ are seen to have roughly the same magnitude and the same density dependence as $M_{\text {Landau }}^{*} / M$. This feature can be understood from the fact that with the dispersion relation of Eqs. (13), (20) and (21) can be reexpressed as

$$
M_{\mathrm{OPT}, \tau}^{*}=\frac{M_{\tau}}{\sqrt{p_{F, \tau}^{2}+M_{\tau}^{2}}} M_{\mathrm{Landau}, \tau}^{*}
$$

and

$$
M_{\mathrm{SOD}, \tau}^{*}=M_{\tau}\left[\frac{M_{\mathrm{Landau}, \tau}^{*}}{E_{\tau}}+\frac{E_{\tau}^{2}-\left(p_{F, \tau}^{2}+M_{\tau}^{2}\right)}{2 E_{\tau}^{2}}\right],
$$



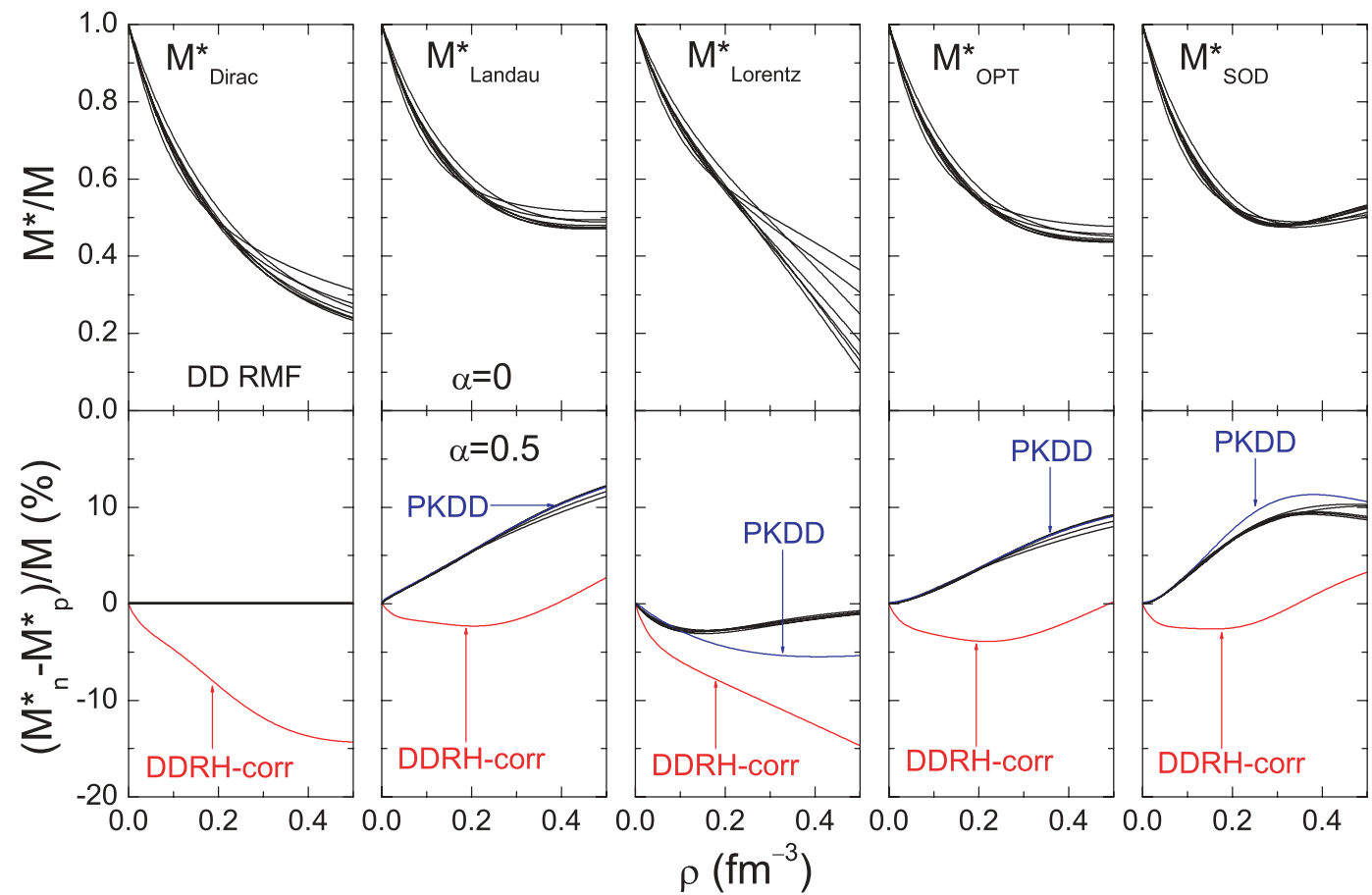

FIG. 9. (Color online) Same as Fig. 8, but for TW99, DD-ME1, DD-ME2, PKDD, DD, DD-F, and DDRH-corr in the density-dependent RMF model.

respectively. Since $p_{F, \tau}^{2} \ll M_{\tau}^{2}$ (for example, $p_{F} \approx$ $385 \mathrm{MeV} / c$ at $\rho_{B}=0.5 \mathrm{fm}^{-3}$ ), we have $M_{\tau} / \sqrt{p_{F, \tau}^{2}+M_{\tau}^{2}} \approx 1$ (with an error of a few percent) and thus $M_{\mathrm{OPT}, \tau}^{*} \approx M_{\mathrm{Landau}, \tau}^{*}$. Furthermore, the second term in Eq. (118) can be neglected compared with the first term as $M_{\tau} / E_{\tau} \sim 1$ (it is a good approximation at low densities and with an error of about $20 \%$ at high densities, e.g., $\rho_{B}=0.5 \mathrm{fm}^{-3}$ ). As a result, we have $M_{\mathrm{SOD}, \tau}^{*} \sim M_{\text {Landau }, \tau}^{*}$.

From the Dirac equation, one sees that condensed scalar fields (scalar self-energies) lead to a shift of nucleon mass such that the nuclear matter is described as a system of

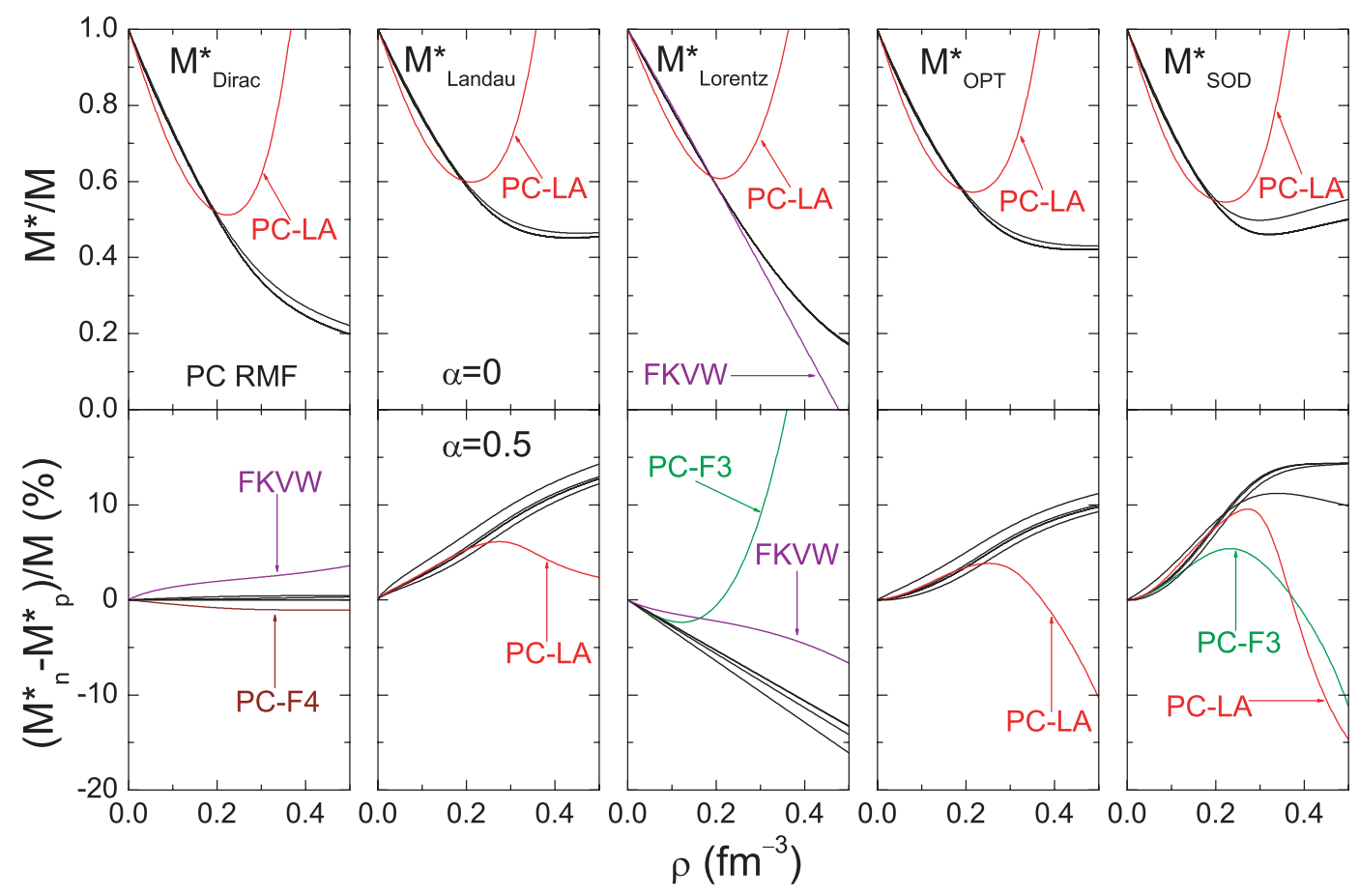

FIG. 10. (Color online) Same as Fig. 8, but for PC-F1, PC-F2, PC-F3, PC-F4, PC-LA, and FKVW in the point-coupling RMF model. 
pseudonucleons with masses $M^{*}$ (Dirac mass) moving in classical vector fields with $\delta$ meson field or isovector-scalar potential further generating the splitting of the proton and neutron Dirac masses in asymmetric nuclear matter. For the isospin splitting of $M_{\text {Dirac }}^{*}$ in neutron-rich nuclear matter, it is interesting to see that the parameter sets HA, NL $\rho \delta$, DDRHcorr, and PC-F4 give $M_{\text {Dirac, } p}^{*}>M_{\text {Dirac, } n}^{*}$ while PC-F2, PCLA, and FKVW exhibit the opposite behavior of $M_{\text {Dirac, } p}^{*}<$ $M_{\text {Dirac }, n}^{*}$. This feature implies that the isospin-dependent scalar potential can be negative or positive depending on the parameter set used. In the nonlinear RMF model, we obtain from Eqs. (32) and (37)

$$
M_{\mathrm{Dirac}, n}^{*}-M_{\mathrm{Dirac}, p}^{*}=-2\left(\frac{g_{\delta}}{m_{\delta}}\right)^{2}\left(\rho_{S, n}-\rho_{S, p}\right),
$$

which indicates that we always have $M_{\text {Dirac }, p}^{*}>M_{\text {Dirac }, n}^{*}$ in the neutron-rich nuclear matter where $\rho_{S, n}>\rho_{S, p}$. This argument is also applicable to the density-dependent RMF model by replacing $g_{\delta}$ with the density-dependent $\Gamma_{\delta}$. For the nonlinear point-coupling models, we have, on the other hand,

$$
M_{\text {Dirac }, n}^{*}-M_{\text {Dirac }, p}^{*}=2 \alpha_{T S}\left(\rho_{S, n}-\rho_{S, p}\right) .
$$

A similar equation can be obtained for the density-dependent point-coupling models with the replacement of $\alpha_{T S}$ by the density-dependent $G_{T S}$. Therefore, the isospin splitting of $M_{\text {Dirac }}^{*}$ in neutron-rich nuclear matter depends on the sign of the isovector-scalar coupling constant $\alpha_{T S}$ and $G_{T S}$ in the point-coupling models. Since the value of $\alpha_{T S}$ in PC-F2 and PC-LA and the value of $G_{T S}$ in FKVW are positive, these parameter sets lead to the isospin splitting $M_{\text {Dirac }, n}^{*}>M_{\text {Dirac, } p}^{*}$ in neutron-rich nuclear matter, which is opposite to that in other parameter sets considered here. The isospin splitting of $M_{\text {Dirac }}^{*}$ is directly related to the isovector spin-orbit potential that determines the isospin-dependent spin-orbit splitting in finite nuclei. Unfortunately, there is no clear experimental indication about the isospin dependence of the spin-orbit splitting in finite nuclei [48], so detailed experimental data on the single-particle energy levels in exotic nuclei are needed to pin down the isospin splitting of $M_{\text {Dirac }}^{*}$ in asymmetric nuclear matter.

For the isospin splitting of $M_{\text {Landau }}^{*}$ in neutron-rich nuclear matter, most parameter sets give $M_{\text {Landau, } n}^{*}>M_{\text {Landau, } p}^{*}$, which is consistent with the usual results in nonrelativistic models. The parameter sets NL $\rho \delta$ and DDRH-corr give, however, the opposite result because of the strong isospin splitting of $M_{\text {Dirac }}^{*}$ with $M_{\text {Dirac }, n}^{*}<M_{\text {Dirac }, p}^{*}$ for NL $\rho \delta$ and DDRH-corr and because $M_{\text {Landau }}^{*}$ is related to the Fermi momentum and $M_{\text {Dirac }}^{*}$ according to Eq. (115). The isospin splitting $M_{\text {Landau }, n}^{*}>$ $M_{\text {Landau, } p}^{*}$ implies that neutrons have a larger level density at the Fermi energy and thus more compressed single-particle levels in finite nuclei than protons.

For the isospin splitting of $M_{\text {Lorentz }}^{*}$ in neutron-rich nuclear matter, all parameter sets give $M_{\text {Lorentz, } p}^{*}>M_{\text {Lorentz, } n}^{*}$, except that PC-L3 gives $M_{\text {Lorentz, } p}^{*}<M_{\text {Lorentz, } n}^{*}$ at high densities. From Eq. (116), we have

$$
M_{\text {Lorentz }, n}^{*}-M_{\text {Lorentz }, p}^{*}=-\left(\Sigma_{n}^{0}-\Sigma_{p}^{0}\right),
$$

which leads to the observed isospin splitting $M_{\text {Lorentz, } p}^{*}>$ $M_{\text {Lorentz, } n}^{*}$ as we generally have $\Sigma_{n}^{0}>\Sigma_{p}^{0}$ as discussed above.
For the parameter set PC-L3, it includes a higher-order isovector-vector term through the parameter $\gamma_{T V}$. Since the latter has a negative value and dominates at high densities according to Eq. (82), it leads to $\Sigma_{n}^{0}<\Sigma_{p}^{0}$ and thus $M_{\text {Lorentz, } p}^{*}<M_{\text {Lorentz, } n}^{*}$ at high densities. The isospin splittings of $M_{\mathrm{OPT}}^{*} / M$ and $M_{\mathrm{SOD}}^{*} / M$ in neutron-rich nuclear matter show a similar behavior as that for $M_{\text {Landau }}^{*}$ as expected from the discussions following Eqs. (117) and (118).

\section{Nucleon scalar density}

The nucleon scalar density as defined in Eq. (34) is the source for the nucleon scalar self-energy (scalar potential). In the RMF model, the isospin-dependent nucleon scalar density is uniquely related to the nucleon Dirac mass as shown in Eq. (35). The latter equation also shows that the scalar density is less than the baryon density due to the factor $M_{i}^{*} / \sqrt{\vec{k}^{2}+\left(M_{i}^{*}\right)^{2}}$ which causes a reduction of the contribution of rapidly moving nucleons to the scalar source term. This mechanism is responsible for nuclear matter saturation in the mean-field theory and essentially distinguishes relativistic models from nonrelativistic ones. In practice, the isospindependent nucleon scalar density is also an essential ingredient for evaluating the relativistic optical potential for neutrons and protons in the relativistic impulse approximation (see, e.g., Refs. [90,93] and references therein).

In Fig. 11, we show the neutron and proton scalar densities as functions of the baryon density $\rho_{B}$ in nuclear matter with

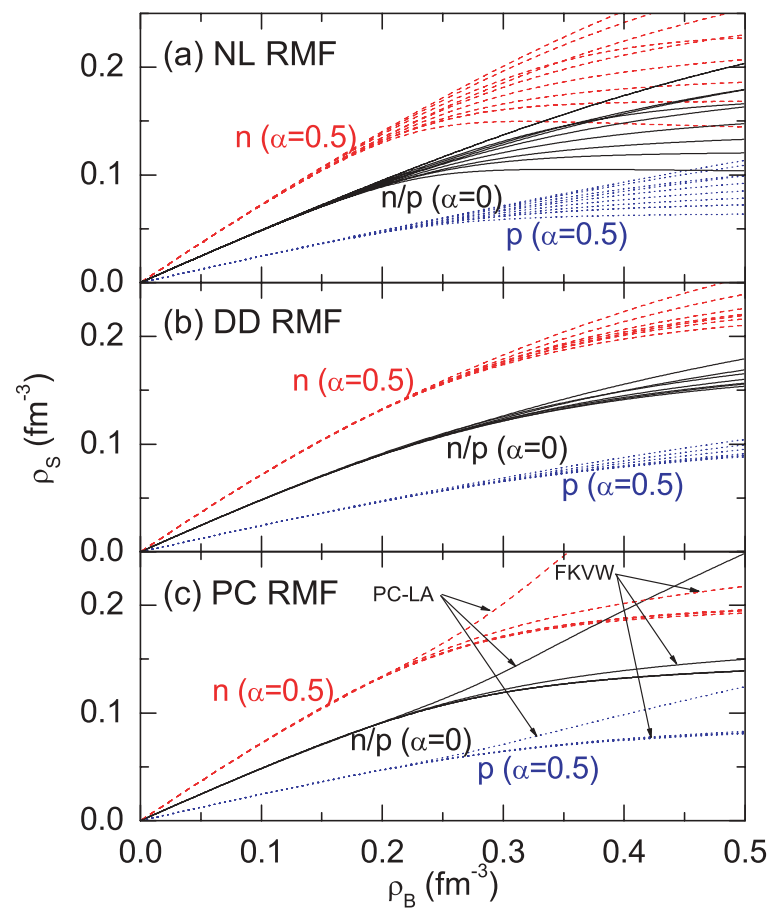

FIG. 11. (Color online) Neutron and proton scalar densities as functions of baryon density in nuclear matter with isospin asymmetry $\alpha=0$ and 0.5 for the parameter sets NL1, NL2, NL3, NL-SH, TM1, PK1, FSU-Gold, HA, NL $\rho$, and NL $\rho \delta$ of the nonlinear RMF model (a); TW99, DD-ME1, DD-ME2, PKDD, DD, DD-F, and DDRH-corr of the density-dependent RMF model (b); PC-F1, PC-F2, PC-F3, PC-F4, PC-LA, and FKVW of the point-coupling RMF model (c). 
isospin asymmetry $\alpha=0$ and 0.5 for the 23 parameter sets from the nonlinear, density-dependent, and point-coupling $\mathrm{RMF}$ models. It is seen that the neutron scalar density is larger than that of protons in neutron-rich nuclear matter at a fixed baryon density. Although results for different parameter sets are almost the same at lower baryon densities, they become different when $\rho_{B} \gtrsim 0.25 \mathrm{fm}^{-3}$, and this is consistent with the conclusions of Refs. [90,93]. In particular, different parameter sets in the nonlinear RMF model predict a larger uncertainty for the value of the nucleon scalar density at high baryon density, while all the parameter sets (except PC-LA) in the density-dependent RMF model and point-coupling models give roughly the same results for the nucleon scalar density. These features are consistent with the results for the density dependence of nucleon Dirac mass shown in Figs. 8-10. At low baryon densities, neutron and proton scalar densities are seen to increase roughly linearly with baryon density, and this can be easily understood from Eq. (35), which is reduced to the following expression at low densities $(|\vec{k}| \rightarrow 0$ due to $\left.k_{F} \rightarrow 0\right)$ :

$$
\begin{aligned}
\rho_{S, i} & \approx \frac{2}{(2 \pi)^{3}} \int_{0}^{k_{F}^{i}} d^{3} k \frac{M_{i}^{*}}{M_{i}^{*}} \\
& =\frac{2}{(2 \pi)^{3}} \int_{0}^{k_{F}^{i}} d^{3} k=\rho_{B, i}, \quad i=p, n .
\end{aligned}
$$

Therefore, neutron and proton scalar densities generally approach their respective baryon densities in asymmetric nuclear matter at low baryon densities.

\section{SUMMARY AND CONCLUSIONS}

Using different versions of relativistic mean-field models that are commonly used in current nuclear structure studies, i.e., the nonlinear model, the model with density-dependent nucleon-meson coupling, and the point-coupling model, we have investigated systematically the isospin-dependent bulk and single-particle properties of isospin-asymmetric nuclear matter. In particular, we considered 23 parameter sets commonly and successfully used in nuclear structure studies, i.e., NL1, NL2, NL3, NL-SH, TM1, PK1, FSU-Gold, HA, $\mathrm{NL} \rho, \mathrm{NL} \rho \delta$ for the nonlinear RMF model; TW99, DD-ME1, DD-ME2, PKDD, DD, DD-F, and DDRH-corr for the densitydependent RMF model; and PC-F1, PC-F2, PC-F3, PC-F4, PC-LA, and FKVW for the point-coupling RMF model. Most of the parameter sets are obtained from fitting the binding energies and charge radii of a large number of nuclei in the periodic table or the results from the microscopic DBHF approach, which have been shown to describe successfully a number of the properties of finite nuclei.

Using these models, we have studied the density dependence of nuclear symmetry energy and compared the results with the symmetry energy recently extracted from the analyses of the isospin diffusion data from heavy-ion collisions based on an isospin- and momentum-dependent transport model with in-medium $N N$ cross sections, the isoscaling analyses of isotope ratios in intermediate energy heavy-ion collisions, and measured isotopic dependence of the giant monopole resonances in even- $A \mathrm{Sn}$ isotopes. These analyses have led to the extraction of $L=88 \pm 25 \mathrm{MeV}$ for the slope parameter of the nuclear symmetry energy at saturation density and $K_{\text {asy }}=-500 \pm 50$ or $-550 \pm 100 \mathrm{MeV}$ for the isospindependent part of the isobaric incompressibility of isospin asymmetric nuclear matter, which may represent the most stringent phenomenological constraints available so far on the nuclear symmetry energy at subsaturation densities. Using these constraints, we have found that among the 23 parameter sets considered in the present work, only six sets, i.e., TM1, NL $\rho$, NL $\rho \delta$, PKDD, PC-LA, and FKVW, have nuclear symmetry energies that are consistent with the extracted $L$ value of $88 \pm 25 \mathrm{MeV}$, while 15 sets, i.e., NL3, NL-SH, TM1, PK1, HA, NL $\rho$, NL $\rho \delta$, TW99, PKDD, DD-F, PC-F1, PC-F2, PC-F3, PC-F4, and FKVW, have nuclear symmetry energies that are consistent with the extracted $K_{\text {asy }}$ value of $-500 \pm 50$ or $-550 \pm 100 \mathrm{MeV}$. Furthermore, we have found surprisingly that only five of the 23 parameter sets, i.e., TM1, NL $\rho$, NL $\rho \delta$, PKDD, and FKVW, have nuclear symmetry energies that are consistent with the extracted values for both $L$ and $K_{\text {asy }}$. We have noted that most parameter sets in the nonlinear and point-coupling RMF models predict stiffer symmetry energies, while those in the density-dependent RMF model give softer symmetry energies. These features are probably related to the rather limited flexibility in the parametrization of the isovector channel in all RMF models and also the fact that most of the parameter sets are obtained from fitting properties of finite nuclei which are mostly near the $\beta$-stability line and thus have less constraint on the isospin-dependent properties of asymmetric nuclear matter. Moreover, we have focused here on the behavior of the symmetry energy around saturation density while the parameter sets in RMF models are fitted to the properties of finite nuclei that are more sensitive to the properties of the nuclear symmetry energy at subsaturation densities.

We have also investigated the energy dependence of three different nucleon optical potentials, i.e., the Schrödingerequivalent potential $U_{\mathrm{SEP}}[\mathrm{Eq}$. (10)], the optical potential from the difference between the total energy of a nucleon in nuclear medium and its energy at the same momentum in free space $U_{\mathrm{OPT}}$ [Eq. (12)], and the optical potential based on the second-order Dirac equation $U_{\text {SOD }}$ [Eq. (15)], as well as their corresponding symmetry potentials $U_{\text {sym }}^{\mathrm{SEP}}, U_{\mathrm{sym}}^{\mathrm{OPT}}$, and $U_{\text {sym }}^{\text {SOD }}$ as functions of momentum. The results indicate that different optical potentials in symmetric nuclear matter exhibit similar energy dependence at low energies but have different high energy behaviors. In particular, at high energies, $U_{\mathrm{SEP}}$ continues to increase linearly with momentum, while $U_{\mathrm{OPT}}$ and $U_{\text {SOD }}$ seem to saturate and thus display a more satisfactory high energy limit compared to the optical potentials extracted from proton-nucleus scatterings using the Dirac phenomenology. On the other hand, the nuclear symmetry potential at a fixed baryon density can increase or decrease with increasing nucleon momentum depending on the definition for the nucleon optical potential and the interactions used. For $U_{\text {sym }}^{\text {SEP }}$ at $\rho_{B}=0.16 \mathrm{fm}^{-3}$, results from all 23 parameter sets show that it increases with momentum, which is consistent with the predictions of microscopic DBHF and chiral perturbation calculations at low momenta (less than about $300 \mathrm{MeV} / \mathrm{c}$ ) but is inconsistent with the experimental result that the nuclear 
symmetry potential at saturation density (the Lane potential $U_{\text {Lane }}$ ) decreases at low energies (beam energy $E_{\text {kin }}$ above 0 and less than about $100 \mathrm{MeV}$ and corresponding momentum values are from about 300 to $470 \mathrm{MeV} / \mathrm{c}$ ) and predictions of the relativistic impulse approximation at higher energies. For $U_{\text {sym }}^{\mathrm{OPT}}$ and $U_{\text {sym }}^{\mathrm{SOD}}$, they can, however, decrease with momentum for some parameter sets, which is qualitatively consistent with the experimental constraint. Again, we emphasize that for the three definitions of the optical potential and thus their corresponding nuclear symmetry potentials, only $U_{\mathrm{SEP}, \tau}$ is well-defined theoretically and is Schrödinger-equivalent, whereas $U_{\mathrm{OPT}, \tau}$ and $U_{\mathrm{SOD}, \tau}$ are used here for reference, because $U_{\mathrm{OPT}, \tau}$ has been extensively used in microscopic DBHF calculations [81] and transport models for heavy-ion collisions [82] and $U_{\mathrm{SOD}, \tau}$ has been used in analyses of the relativistic optical potential based on the Dirac phenomenology [83].

We have further explored different nucleon effective masses, i.e., $M_{\text {Dirac }}^{*}, M_{\text {Landau }}^{*}, M_{\text {Lorentz }}^{*}, M_{\mathrm{OPT}}^{*}$, and $M_{\mathrm{SOD}}^{*}$ in symmetric nuclear matter as well as their isospin splittings in neutron-rich nuclear matter. Most of the parameter sets give reasonable values for $M_{\text {Dirac }}^{*}$ as required by the spinorbit splitting data in finite nuclei but too small values for $M_{\text {Landau, }}^{*}$ implying that they would give too small a level density at the Fermi energy and too large a spread of the single-particle levels in finite nuclei. $M_{\text {Lorentz }}^{*}$ is found to display the strongest (almost linear) density dependence even at high densities. Interestingly, including the isovector-scalar channel leads to the isospin splitting of $M_{\text {Dirac }}^{*}$, and $M_{\text {Dirac }, n}^{*}>$ $M_{\text {Dirac, } p}^{*}$ is always obtained in neutron-rich nuclear matter for the nonlinear and density-dependent RMF models, but an opposite result can be observed in the point-coupling model. For $M_{\text {Landau }}^{*}$, most parameter sets give the isospin splitting $M_{\text {Landau }, n}^{*}>M_{\text {Landau, } p}^{*}$ in neutron-rich nuclear matter, which is consistent with the usual results in nonrelativistic models, while an opposite isospin splitting is observed for $M_{\text {Lorentz }}^{*}$. In addition, $M_{\mathrm{OPT}}^{*}$ and $M_{\mathrm{SOD}}^{*}$ are found to display behaviors similar to that of $M_{\text {Landau }}^{*}$

Finally, we have studied the baryon density dependence of the nucleon scalar density and its isospin splitting in neutronrich nuclear matter. The results indicate that the neutron scalar density is larger than that of proton in neutron-rich nuclear matter at a fixed baryon density. At low baryon densities, the neutron and proton scalar densities generally approach their respective baryon densities in asymmetric nuclear matter.

In the present work, we have focused on three versions of standard RMF models, i.e., the nonlinear, density-dependent, and point-coupling RMF models. We note that some recent works [116-120] have extended the standard RMF models to include density-dependent hadron masses and meson coupling constants via the Brown-Rho (BR) scaling [121]. In particular, the parameter sets SLC and SLCd constructed in Refs. [118,119] not only are consistent with current experimental results for symmetric matter at normal and supranormal densities and the symmetry energy constrained by the isospin diffusion data at subsaturation densities but also give a fairly satisfactory description of the ground state properties of finite nuclei, including binding energies, charge radii, and neutron skin thickness.
In all standard RMF models, the nucleon self-energies are independent of momentum/energy. As a result, the Dirac and Landau masses obtained from these models cannot be simultaneously consistent with experimental data [see, e.g., Eq. (115)]. Also, the Schrödinger-equivalent potential $U_{\text {SEP }}$ [Eq. (10)] in these models increases linearly with nucleon energy even at high energies. Recently, momentum-dependent nucleon self-energies have been introduced in the RMF model by including in the Lagrangian density the couplings of meson fields to the derivatives of nucleon densities [94,122], and the results indicate that a reasonable energy dependence of the Schrödinger-equivalent potential in symmetric nuclear matter at saturation density can be obtained, and the Landau mass can also be increased to a more reasonable value while keeping the Dirac mass unchanged, which further leads to an improved description of $\beta$-decay half-lives of neutron-rich nuclei in the $Z \approx 28$ and $Z \approx 50$ regions [95]. In the framework of densityfunctional theory, including the couplings of meson fields to the derivatives of nucleon densities in the Lagrangian density provides an effective way to take into account higher-order effects. Another way to introduce the momentum dependence in nucleon self-energies is to include the Fock exchange terms by means of the relativistic Hartree-Fock (RHF) approximation, even though in practice the inclusion of the Fock terms would increase significantly the numerical complexity such that it would be very difficult to find appropriate effective Lagrangians for the RHF model to give a satisfactory quantitative description of the nuclear structure properties compared with standard RMF models [74,123-132]. Recently, there have been some developments in the density-dependent RHF approach [133-135]. It has been shown that the density-dependent RHF model can describe the properties of both finite nuclei and nuclear matter with results comparable to those from standard RMF models. A more phenomenological way to improve the results of RMF models is to introduce momentum- as well as isospin-dependent form factors in the meson-nucleon coupling constants. It has been shown in Refs. [136-138] that the empirically observed energy dependence of the nuclear optical potential in symmetric nuclear matter at saturation density can be reproduced by relativistic mean-field models with momentum-dependent form factors. Finally, to better understand the isospin-dependent properties of asymmetric nuclear matter, it is crucial to investigate the density and momentum dependence of the underlying isovector nuclear effective interaction. To reach this ultimate goal, we need not only more advanced theoretical approaches but also more experimental data both on finite nuclei, especially those far from $\beta$-stability line, and from heavy-ion reactions induced by high energy neutron-rich nuclei.

\section{ACKNOWLEDGMENTS}

We would like to thank Paolo Finelli, Wei-Zhou Jiang, Plamen G. Krastev, and Gao-Chan Yong for helpful discussions and communications. L.W.C. also thanks the hospitality of Texas A\&M University at Commerce and College Station where part of the work was done. This work was supported in part by the National Natural Science Foundation of China 
under Grant Nos. 10575071 and 10675082, MOE of China under project NCET-05-0392, Shanghai Rising-Star Program under Grant No. 06QA14024, the SRF for ROCS, SEM of China, the China Major State Basic Research Development Program under Contract No. 2007CB815004 (L.W.C.), the U.S. National Science Foundation under Grant No. PHY0457265, the Welch Foundation under Grant No. A-1358 (C.M.K.), the U.S. National Science Foundation under Grant No. PHY-0652548, and the Research Corporation under Award No. 7123 (B.A.L.).

\section{APPENDIX: ISOSPIN- AND MOMENTUM-DEPENDENT MDI INTERACTION}

The isospin- and momentum-dependent MDI interaction is based on a modified finite-range Gogny effective interaction [25]. In the MDI interaction, the potential energy density $V(\rho, \alpha)$ of an asymmetric nuclear matter at total density $\rho$ and isospin asymmetry $\alpha$ is expressed as $[25,56]$

$$
\begin{aligned}
V(\rho, \alpha)= & \frac{A_{u} \rho_{n} \rho_{p}}{\rho_{0}}+\frac{A_{l}}{2 \rho_{0}}\left(\rho_{n}^{2}+\rho_{p}^{2}\right)+\frac{B}{\sigma+1} \frac{\rho^{\sigma+1}}{\rho_{0}^{\sigma}} \\
& \times\left(1-x \alpha^{2}\right)+\frac{1}{\rho_{0}} \sum_{\tau, \tau^{\prime}} C_{\tau, \tau^{\prime}} \\
& \times \iint d^{3} p d^{3} p^{\prime} \frac{f_{\tau}(\vec{r}, \vec{p}) f_{\tau^{\prime}}\left(\vec{r}, \vec{p}^{\prime}\right)}{1+\left(\vec{p}-\vec{p}^{\prime}\right)^{2} / \Lambda^{2}} .
\end{aligned}
$$

In the mean-field approximation, Eq. (A1) leads to the following single-particle potential for a nucleon with momentum $\vec{p}$ and isospin $\tau$ in asymmetric nuclear matter [25,56]:

$$
\begin{aligned}
U(\rho, \alpha, \vec{p}, \tau) \\
=A_{u}(x) \frac{\rho_{-\tau}}{\rho_{0}}+A_{l}(x) \frac{\rho_{\tau}}{\rho_{0}}+B\left(\frac{\rho}{\rho_{0}}\right)^{\sigma}\left(1-x \alpha^{2}\right) \\
\quad-8 \tau x \frac{B}{\sigma+1} \frac{\rho^{\sigma-1}}{\rho_{0}^{\sigma}} \alpha \rho_{-\tau}+\frac{2 C_{\tau, \tau}}{\rho_{0}} \\
\quad \times \int d^{3} p^{\prime} \frac{f_{\tau}\left(\vec{r}, \vec{p}^{\prime}\right)}{1+\left(\vec{p}-\vec{p}^{\prime}\right)^{2} / \Lambda^{2}}+\frac{2 C_{\tau,-\tau}}{\rho_{0}} \\
\quad \times \int d^{3} p^{\prime} \frac{f_{-\tau}\left(\vec{r}, \vec{p}^{\prime}\right)}{1+\left(\vec{p}-\vec{p}^{\prime}\right)^{2} / \Lambda^{2}}
\end{aligned}
$$

In the above, $\tau=1 / 2(-1 / 2)$ for neutrons (protons); $\sigma=4 / 3 ; f_{\tau}(\vec{r}, \vec{p})$ is the phase-space distribution function at coordinate $\vec{r}$ and momentum $\vec{p}$. The parameters $A_{u}(x), A_{l}(x), B, C_{\tau, \tau}, C_{\tau,-\tau}$, and $\Lambda$ are obtained by fitting the momentum dependence of $U(\rho, \alpha, \vec{p}, \tau)$ to that predicted by the Gogny Hartree-Fock and/or the Brueckner-Hartree-Fock calculations, the saturation properties of symmetric nuclear matter and the symmetry energy of $31.6 \mathrm{MeV}$ at normal nuclear matter density $\rho_{0}=0.16 \mathrm{fm}^{-3}$ [25]. The incompressibility $K_{0}$ of cold symmetric nuclear matter at saturation density $\rho_{0}$ is set to be $211 \mathrm{MeV}$. The parameters $A_{u}(x)$ and $A_{l}(x)$ depend on the $x$ parameter according to

$$
\begin{aligned}
A_{u}(x) & =-95.98-x \frac{2 B}{\sigma+1}, \\
A_{l}(x) & =-120.57+x \frac{2 B}{\sigma+1} .
\end{aligned}
$$

The different $x$ values in the MDI interaction are introduced to vary the density dependence of the nuclear symmetry energy while keeping other properties of the nuclear equation of state fixed [56], and they can be adjusted to mimic the predictions of microscopic and/or phenomenological many-body theories on the density dependence of nuclear matter symmetry energy. The last two terms in Eq. (A2) contain the momentum dependence of the single-particle potential. The momentum dependence of the symmetry potential stems from the different interaction strength parameters $C_{\tau,-\tau}$ and $C_{\tau, \tau}$ for a nucleon of isospin $\tau$ interacting, respectively, with unlike and like nucleons in the background fields. More specifically, we use $C_{\tau,-\tau}=-103.4 \mathrm{MeV}$ and $C_{\tau, \tau}=-11.7 \mathrm{MeV}$.

With $f_{\tau}(\vec{r}, \vec{p})=\frac{2}{h^{3}} \Theta\left(p_{f}(\tau)-p\right)$ for nuclear matter at zero temperature, the integrals in Eqs. (A1) and (A2) can be calculated analytically, and we find

$$
\begin{aligned}
\iint & d^{3} p d^{3} p^{\prime} \frac{f_{\tau}(\vec{r}, \vec{p}) f_{\tau^{\prime}}\left(\vec{r}, \vec{p}^{\prime}\right)}{1+\left(\vec{p}-\vec{p}^{\prime}\right)^{2} / \Lambda^{2}} \\
= & \frac{1}{6}\left(\frac{4 \pi}{h^{3}}\right)^{2} \Lambda^{2}\left\{p_{f}(\tau) p_{f}\left(\tau^{\prime}\right)\left[3\left(p_{f}^{2}(\tau)+p_{f}^{2}\left(\tau^{\prime}\right)\right)-\Lambda^{2}\right]\right. \\
& +4 \Lambda\left[\left(p_{f}^{3}(\tau)-p_{f}^{3}\left(\tau^{\prime}\right)\right) \tan ^{-1} \frac{p_{f}(\tau)-p_{f}\left(\tau^{\prime}\right)}{\Lambda}\right. \\
& \left.-\left(p_{f}^{3}(\tau)+p_{f}^{3}\left(\tau^{\prime}\right)\right) \tan ^{-1} \frac{p_{f}(\tau)+p_{f}\left(\tau^{\prime}\right)}{\Lambda}\right] \\
& +\frac{1}{4}\left[\Lambda^{4}+6 \Lambda^{2}\left(p_{f}^{2}(\tau)+p_{f}^{2}\left(\tau^{\prime}\right)\right)\right. \\
& \left.\left.-3\left(p_{f}^{2}(\tau)-p_{f}^{2}\left(\tau^{\prime}\right)\right)^{2}\right] \ln \frac{\left(p_{f}(\tau)+p_{f}\left(\tau^{\prime}\right)\right)^{2}+\Lambda^{2}}{\left(p_{f}(\tau)-p_{f}\left(\tau^{\prime}\right)\right)^{2}+\Lambda^{2}}\right\}
\end{aligned}
$$

and

$$
\begin{aligned}
\int d^{3} p^{\prime} & \frac{f_{\tau}\left(\vec{r}, \vec{p}^{\prime}\right)}{1+\left(\vec{p}-\vec{p}^{\prime}\right)^{2} / \Lambda^{2}} \\
= & \frac{2}{h^{3}} \pi \Lambda^{3}\left[\frac{p_{f}^{2}(\tau)+\Lambda^{2}-p^{2}}{2 p \Lambda} \ln \frac{\left(p+p_{f}(\tau)\right)^{2}+\Lambda^{2}}{\left(p-p_{f}(\tau)\right)^{2}+\Lambda^{2}}\right. \\
& +\frac{2 p_{f}(\tau)}{\Lambda}-2 \tan ^{-1} \frac{p+p_{f}(\tau)}{\Lambda} \\
& \left.-2 \tan ^{-1} \frac{p-p_{f}(\tau)}{\Lambda}\right] .
\end{aligned}
$$

With these results as well as the well-known contribution from nucleon kinetic energies in the free Fermi gas model, we can thus easily obtain the EOS of asymmetric nuclear matter at zero temperature.

We note that the MDI interaction has been extensively used in the transport model for studying isospin effects in intermediate energy heavy-ion collisions induced by neutronrich nuclei $[26,28,56,139-144]$ and in the study of the thermal properties of asymmetric nuclear matter [145,146]. In particular, the isospin diffusion data from NSCL/MSU have constrained the value of $x$ to between 0 and -1 for nuclear matter densities less than about $1.2 \rho_{0}[56,57]$. 
[1] W. Zhan et al., Int. J. Mod. Phys. E 15, 1941 (2006); http://www.impcas.ac.cn/zhuye/en/htm/247.htm.

[2] Y. Yano, Nucl. Instrum. Methods B 261, 1009 (2007).

[3] See, e.g., http://www.gsi.de/fair/index_e.html.

[4] See, e.g., http://ganinfo.in2p3.fr/research/developments/spiral2.

[5] See, e.g., White Papers of the 2007 NSAC Long Range Plan Town Meeting, Jan. 2007, Chicago, http://dnp.aps.org.

[6] B. A. Li, C. M. Ko, and W. Bauer, Int. J. Mod. Phys. E 7, 147 (1998).

[7] Isospin Physics in Heavy-Ion Collisions at Intermediate Energies, edited by Bao-An Li and W. Udo Schröder (Nova Science, New York, 2001).

[8] P. Danielewicz, R. Lacey, and W. G. Lynch, Science 298, 1592 (2002).

[9] J. M. Lattimer and M. Prakash, Phys. Rep. 333, 121 (2000).

[10] J. M. Lattimer and M. Prakash, Astrophys. J. 550, 426 (2001).

[11] J. M. Lattimer and M. Prakash, Science 304, 536 (2004).

[12] V. Baran, M. Colonna, V. Greco, and M. Di Toro, Phys. Rep. 410, 335 (2005).

[13] A. W. Steiner, M. Prakash, J. M. Lattimer, and P. J. Ellis, Phys. Rep. 411, 325 (2005).

[14] W. D. Myers and W. J. Swiatecki, Nucl. Phys. 81, 1 (1966).

[15] K. Pomorski and J. Dudek, Phys. Rev. C 67, 044316 (2003).

[16] S. Ulrych and H. Müther, Phys. Rev. C 56, 1788 (1997).

[17] E. N. E. van Dalen, C. Fuchs, and A. Faessler, Nucl. Phys. A741, 227 (2004).

[18] Z. Y. Ma, J. Rong, B. Q. Chen, Z. Y. Zhu, and H. Q. Song, Phys. Lett. B604, 170 (2004).

[19] F. Sammarruca, W. Barredo, and P. Krastev, Phys. Rev. C 71, 064306 (2005).

[20] E. N. E. van Dalen, C. Fuchs, and A. Faessler, Phys. Rev. Lett. 95, 022302 (2005).

[21] E. N. E. van Dalen, C. Fuchs, and A. Faessler, Phys. Rev. C 72, 065803 (2005).

[22] J. Rong, Z. Y. Ma, and N. Van Giai, Phys. Rev. C 73, 014614 (2006).

[23] I. Bombaci and U. Lombardo, Phys. Rev. C 44, 1892 (1991).

[24] W. Zuo, L. G. Cao, B. A. Li, U. Lombardo, and C. W. Shen, Phys. Rev. C 72, 014005 (2005).

[25] C. B. Das, S. Das Gupta, C. Gale, and B. A. Li, Phys. Rev. C 67, 034611 (2003).

[26] B. A. Li, C. B. Das, S. Das Gupta, and C. Gale, Phys. Rev. C 69, 011603(R) (2004); Nucl. Phys. A735, 563 (2004).

[27] B. A. Li, Phys. Rev. C 69, 064602 (2004).

[28] L. W. Chen, C. M. Ko, and B. A. Li, Phys. Rev. C 69, 054606 (2004).

[29] J. Rizzo, M. Colonna, M. Di Toro, and V. Greco, Nucl. Phys. A732, 202 (2004).

[30] B. Behera, T. R. Routray, A. Pradhan, S. K. Patra, and P. K. Sahu, Nucl. Phys. A753, 367 (2005).

[31] J. Rizzo, M. Colonna, and M. Di Toro, Phys. Rev. C 72, 064609 (2005).

[32] I. Bombaci, in Isospin Physics in Heavy-Ion Collisions at Intermediate Energies, edited by Bao-An $\mathrm{Li}$ and $\mathrm{W}$. Udo Schröder (Nova Science, New York, 2001), p. 35.

[33] S. Shlomo and D. H. Youngblood, Phys. Rev. C 47, 529 (1993).

[34] D. H. Youngblood, H. L. Clark, and Y.-W. Lui, Phys. Rev. Lett. 82, 691 (1999).
[35] C. Fuchs, Prog. Part. Nucl. Phys. 56, 1 (2006).

[36] J. D. Walecka, Ann. Phys. (NY) 83, 491 (1974).

[37] B. D. Serot and J. D. Walecka, Adv. Nucl. Phys. 16, 1 (1986).

[38] P.-G. Reinhard, Rep. Prog. Phys. 52, 439 (1989).

[39] P. Ring, Prog. Part. Nucl. Phys. 37, 193 (1996).

[40] B. D. Serot and J. D. Walecka, Int. J. Mod. Phys. E 6, 515 (1997).

[41] M. Bender, P.-H. Heenen, and P.-G. Reinhard, Rev. Mod. Phys. 75, 121 (2003).

[42] R. J. Furnstahl, Lect. Notes Phys. 641, 1 (2004).

[43] J. Meng, H. Toki, S. G. Zhoub, S. Q. Zhang, W. H. Long, and L. S. Geng, Prog. Part. Nucl. Phys. 57, 470 (2006).

[44] H. Lenske and C. Fuchs, Phys. Lett. B345, 355 (1995).

[45] C. Fuchs, H. Lenske, and H. H. Wolter, Phys. Rev. C 52, 3043 (1995).

[46] H. Shen, Y. Sugahara, and H. Toki, Phys. Rev. C 55, 1211 (1997).

[47] S. Typel and H. H. Wolter, Nucl. Phys. A656, 331 (1999).

[48] F. Hofmann, C. M. Keil, and H. Lenske, Phys. Rev. C 64, 034314 (2001).

[49] B. A. Nikolaus, T. Hoch, and D. G. Madland, Phys. Rev. C 46, 1757 (1992).

[50] T. Bürvenich, D. G. Madland, J. A. Maruhn, and P.-G. Reinhard, Phys. Rev. C 65, 044308 (2002).

[51] D. G. Madland, T. Bürvenich1, J. A. Maruhn1, and P.-G. Reinhard, Nucl. Phys. A741, 52 (2004).

[52] T. Bürvenich1, D. G. Madland, and P.-G. Reinhard, Nucl. Phys. A744, 92 (2004).

[53] P. Finelli, N. Kaiser, D. Vretenar, and W. Weise, Nucl. Phys. A435, 449 (2004).

[54] P. Finelli, N. Kaiser, D. Vretenar, and W. Weise, Nucl. Phys. A770, 1 (2006).

[55] M. B. Tsang et al., Phys. Rev. Lett. 92, 062701 (2004).

[56] L. W. Chen, C. M. Ko, and B. A. Li, Phys. Rev. Lett. 94, 032701 (2005).

[57] B. A. Li and L. W. Chen, Phys. Rev. C 72, 064611 (2005).

[58] D. V. Shetty, S. J. Yennello, and G. A. Souliotis, Phys. Rev. C 75, 034602 (2007).

[59] T. Li et al., Phys. Rev. Lett. 99, 162503 (2007).

[60] P. J. Siemens, Nucl. Phys. A141, 225 (1970).

[61] O. Sjöberg, Nucl. Phys. A222, 161 (1974).

[62] I. E. Lagaris and V. R. Pandharipande, Nucl. Phys. A369, 470 (1981).

[63] F. S. Zhang and L. W. Chen, Chin. Phys. Lett. 18, 142 (2001).

[64] A. W. Steiner, Phys. Rev. C 74, 045808 (2006).

[65] B. A. Brown, Phys. Rev. Lett. 85, 5296 (2000).

[66] S. Typel and B. A. Brown, Phys. Rev. C 64, 027302 (2001).

[67] C. J. Horowitz and J. Piekarewicz, Phys. Rev. Lett. 86, 5647 (2001); Phys. Rev. C 64, 062802(R) (2001); 66, 055803 (2002).

[68] R. J. Furnstahl, Nucl. Phys. A706, 85 (2002).

[69] S. Karataglidis, K. Amos, B. A. Brown, and P. K. Deb, Phys. Rev. C 65, 044306 (2002).

[70] A. E. L. Dieperink, Y. Dewulf, D. Van Neck, M. Waroquier, and V. Rodin, Phys. Rev. C 68, 064307 (2003).

[71] L. W. Chen, C. M. Ko, and B. A. Li, Phys. Rev. C 72, 064309 (2005). 
[72] A. W. Steiner and B. A. Li, Phys. Rev. C 72, 041601(R) (2005).

[73] M. Prakash and K. S. Bedell, Phys. Rev. C 32, 1118 (1985).

[74] M. Lopez-Quelle, S. Marcos, R. Niembro, A. Bouyssy, and N. V. Giai, Nucl. Phys. A483, 479 (1988).

[75] V. Baran, M. Colonna, M. Di Toro, V. Greco, and M. ZielinskaPfabé, and H. H. Wolter, Nucl. Phys. A703, 603 (2002).

[76] M. M. Sharma, W. T. A. Borghols, S. Brandenburg, S. Crona, A. vander Woude, and M. N. Harakeh, Phys. Rev. C 38, 2562 (1988).

[77] B. G. Todd-Rutel and J. Piekarewicz, Phys. Rev. Lett. 95, 122501 (2005).

[78] M. Jaminon, C. Mahaux, and P. Rochus, Phys. Rev. C 22, 2027 (1980).

[79] M. Jaminon and C. Mahaux, Phys. Rev. C 40, 354 (1989).

[80] H. Feldmeier and J. Lindner, Z. Phys. A 341, 83 (1991).

[81] G. Q. Li and R. Machleidt, Phys. Rev. C 48, 2707 (1993).

[82] P. Danielewicz, Nucl. Phys. A673, 375 (2000).

[83] S. Hama, B. C. Clark, E. D. Cooper, H. S. Sherif, and R. L. Mercer, Phys. Rev. C 41, 2737 (1990).

[84] G. R. Satchler, in Isospin in Nuclear Physics, edited by D. H. Wilkinson (North-Holland, Amsterdam, 1969), p. 391.

[85] G. W. Hoffmann and W. R. Coker, Phys. Rev. Lett. 29, 227 (1972).

[86] P. E. Hodgson, in The Nucleon Optical Model (World Scientific, Singapore, 1994), p. 613.

[87] A. J. Koning and J. P. Delarocje, Nucl. Phys. A713, 231 (2003).

[88] A. M. Lane, Nucl. Phys. 35, 676 (1962).

[89] S. Fritsch, N. Kaiser, and W. Weise, Nucl. Phys. A750, 259 (2005).

[90] L. W. Chen, C. M. Ko, and B. A. Li, Phys. Rev. C 72, 064606 (2005).

[91] C. J. Horowitz, Phys. Rev. C 31, 1340 (1985).

[92] D. P. Murdock and C. J. Horowitz, Phys. Rev. C 35, 1442 (1987).

[93] Z. H. Li, L. W. Chen, C. M. Ko, B. A. Li, and H. R. Ma, Phys. Rev. C 74, 044613 (2006).

[94] S. Typel, Phys. Rev. C 71, 064301 (2005).

[95] T. Marketin, D. Vretenar, and P. Ring, Phys. Rev. C 75, 024304 (2007).

[96] E. Chabanat, P. Bonche, P. Haensel, J. Meyer, and R. Schaeffer, Nucl. Phys. A627, 710 (1997).

[97] E. Chabanat, P. Bonche, P. Haensel, J. Meyer, and R. Schaeffer, Nucl. Phys. A635, 231 (1998).

[98] P.-G. Reinhard, Nucl. Phys. A649, 305c (1999).

[99] D. Lunney, J. M. Pearson, and C. Thibault, Rev. Mod. Phys. 75, 1021 (2003).

[100] H. Müller and B. D. Serot, Nucl. Phys. A606, 508 (1996).

[101] B. Liu, V. Greco, V. Baran, M. Colonna, and M. Di Toro, Phys. Rev. C 65, 045201 (2002).

[102] S. Kubis and M. Kutschera, Phys. Lett. B399, 191 (1997).

[103] S.-J. Lee, J. Fink, A. B. Balantekin et al., Phys. Rev. Lett. 57, 2916 (1986).

[104] G. A. Lalazissis, J. Konig, and P. Ring, Phys. Rev. C 55, 540 (1997).

[105] M. M. Sharma, M. A. Nagarajan, and P. Ring, Phys. Lett. B312, 377 (1993).

[106] Y. Sugahara and H. Toki, Nucl. Phys. A579, 557 (1994).
[107] W. H. Long, J. Meng, N. Van Giai, and S. G. Zhou, Phys. Rev. C 69, 034319 (2004).

[108] J. K. Bunta and S. Gmuca, Phys. Rev. C 68, 054318 (2003).

[109] T. Niksic, D. Vretenar, P. Finelli, and P. Ring, Phys. Rev. C 66 , 024306 (2002).

[110] G. A. Lalazissis, T. Niksic, D. Vretenar, and P. Ring, Phys. Rev. C 71, 024312 (2005).

[111] T. Klahn et al., Phys. Rev. C 74, 035802 (2006).

[112] T. Gaitanos, M. Di Toro, S. Type, V. Baran, C. Fuchs, V. Greco, and H. H. Wolter, Nucl. Phys. A732, 24 (2004).

[113] E. D. Cooper, S. Hama, B. C. Clark, and R. L. Mercer, Phys. Rev. C 47, 297 (1993).

[114] H. F. Arellano and H. V. von Geramb, Phys. Rev. C 66, 024602 (2002).

[115] C. Fuchs and H. H. Wolter, Eur. Phys. J. A 30, 5 (2006).

[116] B. Liu, H. Guo, V. Greco, U. Lombardo, M. Di Toro, and Cai-Dian Lu, Eur. Phys. J. A 22, 337 (2004).

[117] S. S. Avancini and D. P. Menezes, Phys. Rev. C 74, 015201 (2006).

[118] W. Z. Jiang, B. A. Li, and L. W. Chen, Phys. Lett. B653, 184 (2007).

[119] W. Z. Jiang, B. A. Li, and L. W. Chen, Phys Rev. C 76, 054314 (2007)

[120] A. S. Khvorostukhin, V. D. Toneev, and D. N. Voskresensky, Nucl. Phys. A791, 180 (2007).

[121] G. E. Brown and M. Rho, Phys. Rev. Lett. 66, 2720 (1991).

[122] S. Typel, T. v. Chossy, and H. H. Wolter, Phys. Rev. C 67, 034002 (2003).

[123] L. D. Miller, Phys. Rev. C 9, 537 (1974).

[124] R. Brockmann, Phys. Rev. C 18, 1510 (1978).

[125] M. Jaminon, C. Mahaux, and P. Rochus, Nucl. Phys. A365, 371 (1981).

[126] C. J. Horowitz and B. D. Serot, Nucl. Phys. A399, 529 (1983).

[127] P. G. Blunden and M. J. Iqbal, Phys. Lett. B196, 295 (1987).

[128] A. Bouyssy, J.-F. Mathiot, N. Van Giai, and S. Marcos, Phys. Rev. C 36, 380 (1987).

[129] P. Bernardos, V. N. Fomenko, N. V. Giai, M. L. Quelle, S. Marcos, R. Niembro, and L. N. Savushkin, Phys. Rev. C 48, 2665 (1993).

[130] R. Niembro, P. Bernardos, M. Lopez-Quelle, and S. Marcos, Phys. Rev. C 64, 055802 (2001).

[131] S. Marcos, L. N. Savushkin, V. N. Fomenko, M. López-Quelle, and R. Niembro, J. Phys. G 30, 703 (2004).

[132] M. Lopez-Quelle, L. N. Savushkin, S. Marcos, and R. Niembro, J. Phys. G 30, 703 (2004).

[133] W. H. Long, N. Van Giai, and J. Meng, Phys. Lett. B640, 150 (2006)

[134] W. H. Long, H. Sagawa, J. Meng, and N. Van Giai, Phys. Lett. B639, 242 (2006).

[135] W. H. Long, H. Sagawa, N. Van Giai, and J. Meng, Phys. Rev. C 76, 034314 (2007).

[136] K. Weber, B. Blättel, W. Cassing, H. C. Dönges, V. Koch, A. Lang, and U. Mosel, Nucl. Phys. A539, 713 (1992).

[137] T. Maruyama, W. Cassing, U. Mosel, S. Teis, and K. Weber, Nucl. Phys. A573, 653 (1994).

[138] P. K. Sahu, W. Cassing, U. Mosel, and A. Ohnishi, Nucl. Phys. A672, 376 (2000).

[139] B. A. Li, G. C. Yong, and W. Zuo, Phys. Rev. C 71, 014608 (2005).

[140] B. A. Li, G. C. Yong, and W. Zuo, Phys. Rev. C 71, 044604 (2005). 
[141] B. A. Li, L. W. Chen, G. C. Yong, and W. Zuo, Phys. Lett. B634, 378 (2006).

[142] G. C. Yong, B. A. Li, L. W. Chen, and W. Zuo, Phys. Rev. C 73, 034603 (2006).

[143] G. C. Yong, B. A. Li, and L. W. Chen, Phys. Rev. C 74, 064617 (2006).
[144] G. C. Yong, B. A. Li, and L. W. Chen, Phys. Lett. B650, 344 (2007).

[145] J. Xu, L. W. Chen, B. A. Li, and H. R. Ma, Phys. Rev. C 75, 014607 (2007).

[146] J. Xu, L. W. Chen, B. A. Li, and H. R. Ma, Phys. Lett. B650, 348 (2007). 